\title{
A Novel Distributed Privacy Paradigm for Visual Sensor Networks Based on Sharing Dynamical Systems
}

\author{
William Luh, Deepa Kundur, and Takis Zourntos \\ Department of Electrical and Computer Engineering, 214 Zachry Engineering Center, Texas A\&M University, College Station, \\ TX 77843-3128, USA
}

Received 5 January 2006; Revised 29 April 2006; Accepted 30 April 2006

Recommended by Chun-Shien Lu

Visual sensor networks (VSNs) provide surveillance images/video which must be protected from eavesdropping and tampering en route to the base station. In the spirit of sensor networks, we propose a novel paradigm for securing privacy and confidentiality in a distributed manner. Our paradigm is based on the control of dynamical systems, which we show is well suited for VSNs due to its low complexity in terms of processing and communication, while achieving robustness to both unintentional noise and intentional attacks as long as a small subset of nodes are affected. We also present a low complexity algorithm called TANGRAM to demonstrate the feasibility of applying our novel paradigm to VSNs. We present and discuss simulation results of TANGRAM.

Copyright @ 2007 Hindawi Publishing Corporation. All rights reserved.

\section{INTRODUCTION}

Visual data is an integral part of the interface between humans and their environment. Visual data in the form of images and video can be used to enhance a human operator's ability to reliably make crucial decisions in the face of alerts provided by sensing mechanisms. For example, in a combat field, a sensor network can be deployed to sense temperature, toxins, vibrations/movement, and so forth. To reliably assess whether a change in the sensed phenomena is due to enemy infiltration or natural environmental and fauna causes, it is useful to obtain additional side information in the form of an image. As another example, in health care facilities $[1,2]$, one may measure a patient's vital statistics, such as heart rate, using sensors. When such measured statistics indicate that the patient is in imminent danger, visual side information may quickly determine whether the measurements are valid or caused by misplaced or malfunctioning sensors. Following this motivation, acquisition of visual data in sensor networks can be used to enhance the quality of service in surveillance applications in which a human operator interfaces at the sink of the network [3]. Such sensor networks are called visual sensor networks (VSNs) or often multimedia sensor networks [4]. The emergence of low-cost portable off-the-shelf sensor devices has thrust forward the development of VSN architectures, systems, and testbeds [13, 5-12].
Acquisition and processing of visual data in sensor networks come at a cost. First, visual data in the form of images or video require larger storage and transmission resources than do traditional scalar data such as temperature or heart rate. These resource requirements are further bloated when every sensor is equipped to acquire and process images and video. Furthermore, image processing requires more power to process than conventional scalar data, and hence VSNs may not meet the resource constraints placed upon traditional scalar-data-based sensor networks. This suggests that visual data should be acquired and processed judiciously, perhaps by one or two cameras within a confined area. However as we consider in this paper, from the perspective of resilience to physical and electronic attack, dense VSNs demonstrate potential for security and surveillance applications.

Visual data may be intercepted by illicit parties for use not originally intended. For example, in a military scenario, interception of surveillance images can be used by an enemy to learn and counter the efforts of a mission. In a health-care scenario, interception of images by outsiders compromises patient privacy rights. Therefore a means to protect these images needs to be built into VSNs. In order to combat physical attacks, electronic means are often employed in addition to more robust ad hoc networking architectures. For example, the "one camera" architecture suggested above is vulnerable to physical attacks such as unlawful interception, tampering, 
or capture of entities in the network. Instead of preventing such actions, it is more feasible to engineer information security mechanisms to deny illicit parties access to the semantic content of visual data.

Traditionally, to deny third parties access to content, encryption is employed [13]. However, recently it has been noted that the use of these powerful cryptosystems on visual data further exacerbates processing power (resource) requirements as mentioned above [14-16]. Measures have been taken to trade off security with processing complexity, and in this paper we follow this philosophy.

In the spirit of sensor networks, we opt for a densely distributed architecture in which every sensor is equipped with a camera for visual acquisition, as well as some simple image processing capabilities. It is not difficult to envision that physical security based on a distributed infrastructure shares all the same advantages as those in which sensor network research pioneers were drawn to. The principle of redundancy compensates for sensor failure either due to natural (i.e., battery failure, noise in the environment, or sensor hardware) or malicious causes. From a physical security standpoint, distribution offers safeguard against illicit capture of a few sensor nodes and its cryptographic keys stored on-board [17, 18], which we term node capture. Attackers are hence forced to capture all nodes or intercept all relevant node communique in order to access semantic information. In this paper, we propose a general paradigm for distributing security in a visual sensor network.

\subsection{Scope and contribution}

We focus, in this paper, on presenting a novel distributed approach to protect dense VSNs against eavesdropping attacks. Other security issues including authentication, message freshness and replay, key management, physical and actuation attacks, and common denial-of-service attacks are, in part, considered by existing sensor network security literature and are beyond the scope of the paper.

In [19], security for the IP-based video surveillance problem is considered. In this paper we consider a distributed security scheme for VSNs in which camera nodes work together. We consider a VSN, which we define to be a collection of sensor nodes each having image acquisition and processing capabilities. Within a VSN, a cluster of nodes is defined to be a subset of $N$ nodes that are recording or capturing the same scene at approximately the same camera orientation. A security goal of each node in a cluster is to send partial visual information, which we call shares to a base station or multiple base stations, such that

(1) the base station(s) can reconstruct (or decrypt) an approximation of the scene being recorded by the cluster when $t+1$ or more shares are available;

(2) interception of $t$ or fewer shares will not reveal the scene being recorded.

Secret sharing, popularized by Shamir [18], is the process by which a trusted central authority called the dealer creates and securely distributes $N$ shares to $N$ participants, such that only certain subsets of participants can recover a secret key $K$ by amalgamating their shares. Our problem is similar to secret sharing, except for the following differences:

(1) traditional secret sharing requires a trusted central authority to create the shares and distribute them securely; in our distributed VSN problem, a different share is created by each node of a cluster (with some minor coordination to guarantee robust recovery);

(2) we allow for $t$ or fewer nodes to be captured, thus revealing any secret keys;

(3) we allow for $t$ or fewer shares to be corrupted or tampered with.

We now point out other existing secret sharing works, and show how our work differs. In visual secret sharing (VSS) [20-23], the goal is similar; a dealer creates $N$ transparencies and securely distributes them to $N$ participants. If a subset of transparencies are overlaid upon one another, the secret image is revealed - this is the decryption process. In most VSS schemes, the decryption process (i.e., overlaying transparencies) has lower complexity than either creating the shares or transmitting/storing them. This is due to the fact that extra information must be embedded into the transparencies in order to support such trivial decryption. In sensor networks, this is not favorable; instead the opposite role is favored, being that complexity is lower at the encrypting end (node end), and higher at the decrypting end (base station end) [24]. Of course VSS also suffers from the need for a trusted central authority as does secret sharing. Next, we point out a distributed encryption scheme that does not require a trusted central authority based on the RSA public-key cryptosystem $[25,26]$. One draw-back of $[25,26]$ is that this public-key cryptography scheme was not developed for sensor networks (but rather for fault-tolerant distributed systems), hence the complexity at the node end is much higher. In addition, the method is not always immune to node capture, particularly if the so-called source node is captured, key information can be used to reveal the secret.

To this end, this paper offers a general paradigm for creating shares in a VSN, and hence many algorithms can be created based on the same principles. The use of dynamic system theory for this purpose is novel and, as we demonstrate, provides the following attractive characteristics: (a) dynamism and evolution to exploit the distributed and collaborative nature of VSNs for share generation, (b) robustness to compensate for sensor error and malicious tampering, (c) obfuscation in order to provide image/video scrambling with a more competitive compromise between security and practicality for VSNs, and (d) flexibility and simplicity for lightweight implementation. The dynamic system approach allows the creative incorporation of the many competing VSN objectives of robustness, security and practicality into a framework with well-developed mathematical background based on Lyapunov theory. This has the advantage of producing a solution that is distributed and lower complexity and hence most appropriate for VSNs in comparison to the existing methods surveyed above. We analyze our proposed technique, called 
TANGRAM, to demonstrate its potential performance and practicality.

Finally, we note that our paradigm is geared towards visual data, or any kind of data whose semantics are not destroyed by small perturbations. The inherent redundant nature of visual data offers both pros and cons. On the one hand, visual redundancy offers resiliency against errors. On the other hand, visual redundancy translates into higher communication and storage costs. Hence a tradeoff between robustness and compression is also considered in this paper.

This paper is separated into three parts. In Section 2, the general paradigm is presented. Within this section we formulate the problem, introduce notations and definitions, and finally present the general architecture and guiding principles used to design a solution. In Section 3, we present an algorithm using our paradigm. In Section 4, we present simulation results to verify visual security.

\section{GENERAL PARADIGM}

In this section we develop the general paradigm. ${ }^{1}$ First we formally present the problem and assumptions. Next we define basic elements used in the framework, and finally we present the paradigm.

\subsection{Problem formulation}

Suppose a collection of $N$ sensor nodes equipped with image acquisition and processing capabilities is deployed in close physical proximity such that they all capture the same scene. To quantify this statement, let $\left\{I_{0}, I_{1}, \ldots, I_{N-1}\right\}$ represent the $N$ grayscale images captured by the $N$ nodes. ${ }^{2}$ Here $I_{i}, 0 \leq i \leq N-1$ are $m \times n$ matrices containing integer values in the set $\{0,1, \ldots, 255\}$. Assume that these $N$ images are noisy versions of a representative image $I_{r}$, such that $I_{i}=I_{r}+n_{r}$, where $n_{r}$ is a random matrix of integers based on some distribution with zero-mean and small variance. This assumption allows us to approximate the different sensor acquisitions as the same image, that is, $I_{i} \approx I_{r}$, which will simplify the computations. We will justify this assumption soon in the coming section.

We also assume that the VSN is capable of pairwise (between two neighboring nodes) and individual (between node and base station) key distributions. In addition, each node is capable of communications to neighboring nodes and to the base station (possibly via multihop networking).

\subsubsection{Goals of this paradigm}

The goal is for each sensor node $i$ to encrypt its image $I_{i}$, resulting in the share $\hat{I}_{i}$, such that for some subset of shares

\footnotetext{
${ }^{1}$ In this paper we generalize the paradigm previously developed in [27], hence encompassing a broader class of algorithms.

2 Color images can be treated in the same manner by defining accompanying color planes dependent on formats such as RGB, HSV, YCbCr, and so forth.
}

$S \subseteq\left\{\hat{I}_{0}, \hat{I}_{1}, \ldots, \hat{I}_{N-1}\right\}$, and subset of sensor nodes ${ }^{3} T \subseteq\{0,1$, $\ldots, N-1\}$ corresponding to $S$

(1) if the cardinality of $S$ is greater than $t$, that is, $|S|>t$, then a visual approximation of $I_{r}$ can be derived from $S$;

(2) if $|S| \leq t$, then a visual approximation of $I_{r}$ cannot be derived from $S$ alone;

(3) if the sensor nodes of $T$ with $|T| \leq t$ are physically captured and removed from the network, any statically stored information ${ }^{4}$ on these nodes along with the corresponding shares in $S$ will not help the attacker in deriving a visual approximation of $I_{r}$;

(4) key management in the form of rekeying or key updates is not necessary to deal with the particular issues addressed in this paper.

We will define the quantitative notion of visual approximation in the coming subsection. Also we note that although existing secret sharing algorithms can be adjusted to satisfy point 3 through rekeying or key updating, our paradigm does not explicitly require key management leading to a more practical solution for distributed VSNs.

\subsubsection{Assumptions}

We now impose the following assumptions on the attacker.

(1) The attacker has limited ability to employ physical attacks on the observation area. Specifically, the attacker cannot deploy his own cameras to capture the same scene as the VSN nodes nor can he physically attack the observation area such as block the scene. ${ }^{5}$ In addition, the attacker can only physically capture and remove nodes from the network, but cannot wiretap a node and eavesdrop on all activities on-board a sensor node.

(2) The attacker can only intercept or tamper the shares of a subset of nodes of cardinality $\leq t$.

(3) The attacker is less likely to intercept communication between nodes without being detected due to the nodes being in close proximity; the attacker is more likely to intercept communication between nodes and the base station(s). See Figure 1.

We impose the following assumptions on the sensor nodes.

(1) The VSN is aware when a node is removed from the network, and will stop communicating with the rogue node.

(2) Every node can perform the duties of any other node. Hence when a node dies, the nodes within a cluster can reorganize their logistics. In addition, nodes are

\footnotetext{
${ }^{3}$ In this paper, we denote $N$ nodes by giving them unique integer identifiers starting with 0 .

${ }^{4}$ By statically stored, we mean keys, codebooks, and so forth. Any values created from computation would not be statically stored.

${ }^{5}$ Physical actuation attacks considered in, for example, [28] are beyond the scope of this work.
} 
replenished so that an area of observation is never left starved for nodes.

(3) We assume that the nodes are capable of repositioning collectively (i.e., rotating panoramically) to capture different scenes in order to avoid the message resend attack [29].

\subsection{Preliminaries}

In this section we define the basic elements used in our paradigm. First we define an image space by converting an image matrix $I_{i}$ into a $(m \cdot n) \times 1$ column vector $\mathbf{x}_{i}$, via a column-wise raster scan as shown in (1).

$I_{i}=\left(\begin{array}{ccccc}x_{1,1} & x_{1,2} & \cdots & x_{1, n-1} & x_{1, n} \\ x_{2,1} & x_{2,2} & \cdots & x_{2, n-1} & x_{2, n} \\ \vdots & \vdots & \ddots & \vdots & \vdots \\ x_{m-1,1} & x_{m-1,2} & \cdots & x_{m-1, n-1} & x_{m-1, n} \\ x_{m, 1} & x_{m, 2} & \cdots & x_{m, n-1} & x_{m, n}\end{array}\right)$,

$\mathbf{x}_{i}=\left(\begin{array}{llllllllll}x_{1,1} & x_{2,1} & \cdots & x_{m, 1} & x_{1,2} & x_{2,2} & \cdots & x_{m, 2} & \cdots & x_{m, n}\end{array}\right)^{T}$.

The collection of all $(m \cdot n) \times 1$ column vectors constitutes our image space, and each column vector is called a state. We note that although we defined an image to take on integer values in the range 0 to 255 , the image space includes all $(m \cdot n) \times 1$ column vectors with real elements. This collection along with the usual operators is a vector space over the real field.

Our notion of visual approximation is based on the norm of a vector. ${ }^{6}$ The norm of a vector $\mathbf{x}_{i}$ is the $l^{2}$-norm denoted by $\left\|\mathbf{x}_{i}\right\|$. To quantify visual similarity or dissimilarity for practical use, two variables $\rho_{0} \leq \rho_{1}$ are chosen as a function of the (image) state $\mathbf{x}_{i}$ in question, such that the annulus centered about $\mathbf{x}_{i}$, as given in (2), completely defines the visual similarity and dissimilarity:

$$
A_{\mathbf{x}_{i}}=\left\{\mathbf{x}: \rho_{0} \leq\left\|\mathbf{x}-\mathbf{x}_{i}\right\| \leq \rho_{1}\right\}
$$

The complement of the annulus can be separated into two regions: the region enclosed by the annulus is called the similar region, where the states in here share the semantics of $\mathbf{x}_{i}$; the region outside the annulus is called the dissimilar region, where the semantics of $\mathbf{x}_{i}$ cannot be visually deduced from states in here. The annulus itself defines a fuzzy region, which accounts for differences in individual perception. Figure 2 illustrates the partitioning of the image space into

\footnotetext{
${ }^{6}$ We note that although this notion does not model the human visual system accurately, it is used often for reasons of simplicity in MPEG encoding, for example block matching [30].
}

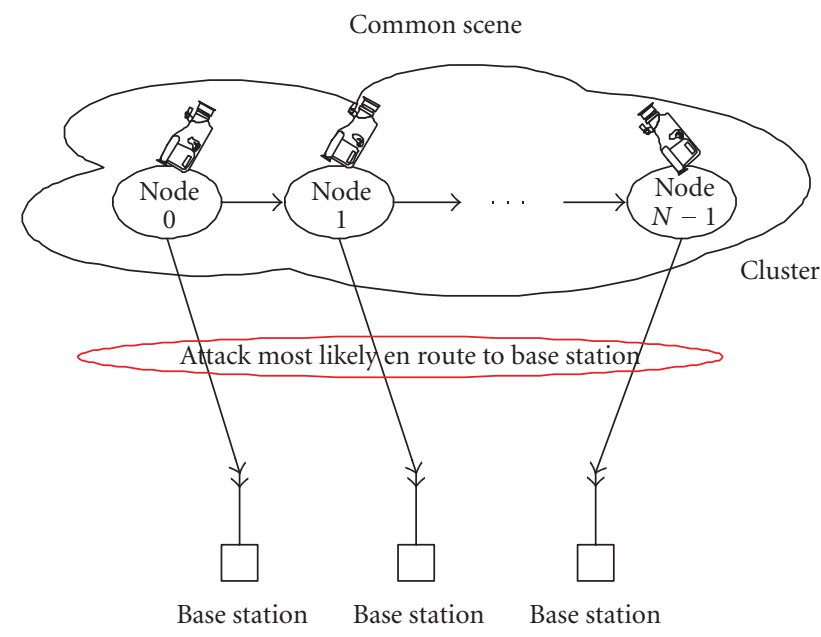

FIGURE 1: Communication scheme, layout of a cluster, and the most common point of attack.

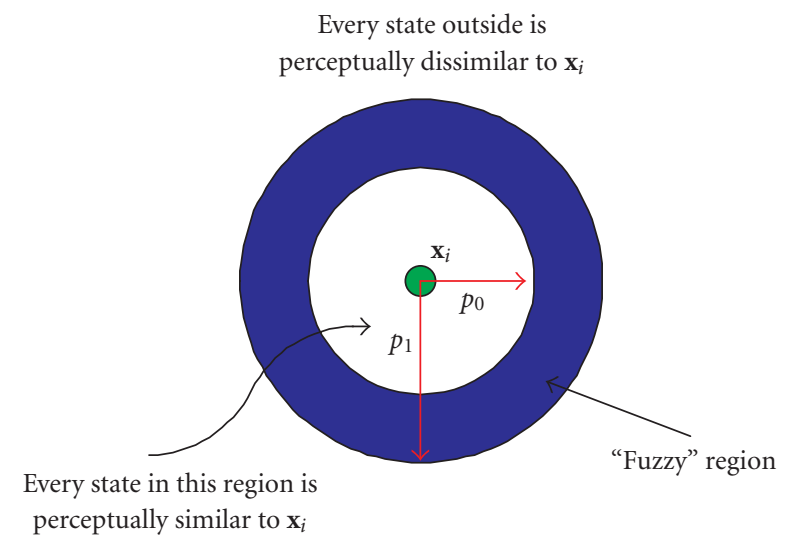

FIGURE 2: Separation of image space into perceptual similar, dissimilar, and fuzzy regions.

similar, dissimilar, and fuzzy regions. It is clear that the variables $\rho_{0} \leq \rho_{1}$ depend on the human visual system, and hence is application-dependent.

\subsection{Architecture and principles}

Suppose that every sensor node records the representative image $I_{r}$ (i.e., the common scene), which corresponds with the representative state $\overline{\mathbf{x}}$. The central idea behind our paradigm is that we design a discrete-time dynamical system such that each node has an access to only a certain part of this dynamical system and not to its entirety. Identifiers 0 to $N-1$ are assigned to each of the $N$ nodes. A node with identifier $k$ is then responsible for applying a control to move the state at time $k$ of the dynamical system closer to the desired $\overline{\mathbf{x}}$. The node's control is the node's share. Since an attacker who intercepts a subset of shares and/or physically captures 


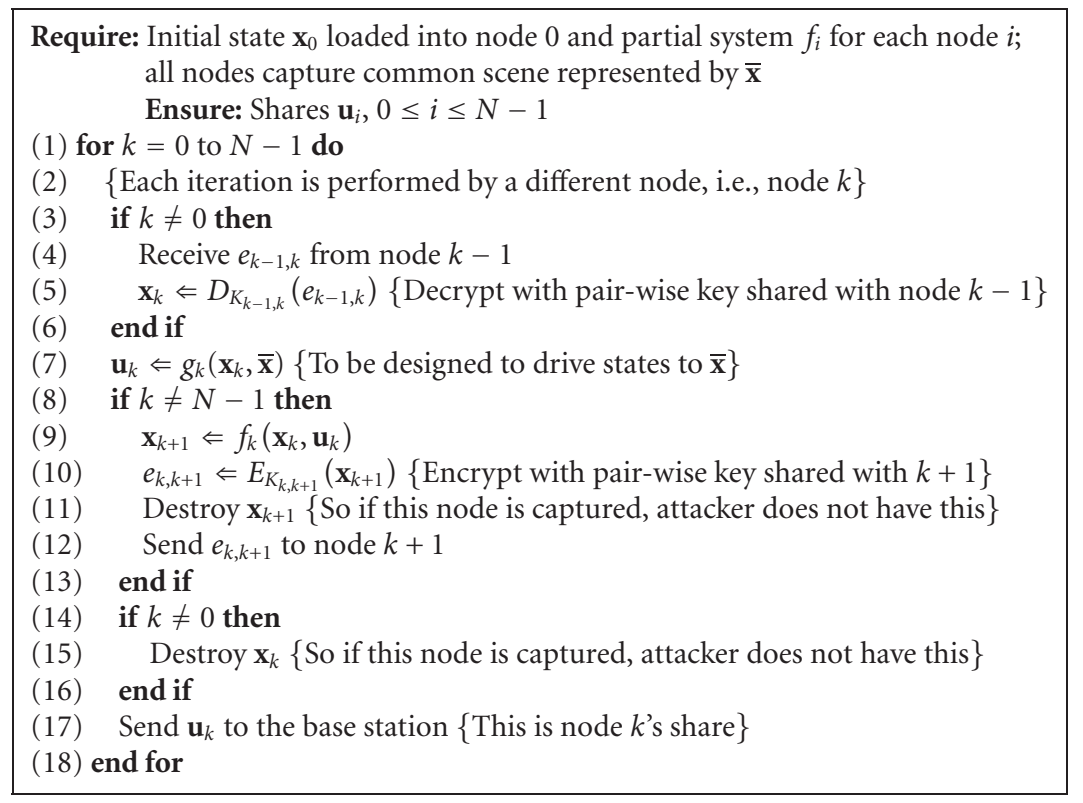

Algorithm 1: Distributed encryption for VSN.

a subset of nodes only knows part of the dynamical system, ${ }^{7}$ the attacker cannot drive this partial dynamical system to the secret $\overline{\mathbf{x}}$. In Section 2.4, we will discuss the motivation for using this paradigm.

From the dynamical systems literature, let $\Sigma_{p}$ be a userdesigned plant that is described by a state equation (discretetime difference equation) as in (3):

$$
\Sigma_{p}: \mathbf{x}_{k+1}=f_{k}\left(\mathbf{x}_{k}, \mathbf{u}_{k}, \mathbf{w}_{k}\right)
$$

Here, the vectors denoted by $\mathbf{x}_{k}$ are called states, the vectors denoted by $\mathbf{u}_{k}$ are external controls/inputs, and $\mathbf{w}_{k}$ is a random vector noise term. Every node agrees ahead of time on a starting node, also called the source node [25], which contains a randomly generated initial state $\mathbf{x}_{0}$ (i.e., independent of $I_{r}$ ), either through preprogramming the node hardware, or some key distribution protocol.

Next we ensure that every node is endowed with only part of the plant or that there is a random component of which only one node is aware. For example, if the system is timevarying, each node is endowed with a unique set of the parameters corresponding to each time instance. To be more precise, let the nodes be numbered 0 to $N-1$. Then node $i$ is endowed with $f_{i}$, and for node $i \neq j, f_{i} \neq f_{j}$; that is each node only knows part of the system. Finally each node runs an optimization algorithm whose goal is to drive any given state to $\overline{\mathbf{x}}$. The pseudocode is presented in the table entitled

\footnotetext{
7 This does not violate Kerckhoff's principle [13], which states that the security of a system should only reside in the key, while the system can be known. In practice, we can publish the system to be used, but keep secret the system parameters, which can be regarded as keys.
}

Algorithm 1. Here we define $e_{i, j}$ to be the encrypted state, which is created by node $i$ and sent to node $j$.

In Algorithm 1, each iteration of the loop reflects the activity of one particular node, namely the node associated with the loop index $k$. We see that the source node 0 starts off the algorithm by applying a function $g_{0}$ (line 7 - to be designed) on the initial state $\mathbf{x}_{0}$ and the representative state $\overline{\mathbf{x}}$ - this is the external input or the so-called control to the plant $\Sigma_{p}$. This control is then applied to the plant (line 9), and the result is encrypted using a pair-wise key shared with node 1 (line 10), and sent to node 1 (line 12). Continuing with the remaining iterations of the loop, each node hereafter receives an encrypted state (line 4 ) from the previous node, which is able to decrypt with its pair-wise key shared with the previous node (line 5). The node then uses this state to derive a new control (line 7), which drives the states closer to the desired representative state $\overline{\mathbf{x}}$. The new state generated by this control (line 9) is then encrypted with the pair-wise key shared with the next node (line 10) and sent to the next node (line 12). The controls generated by each node constitute the set of shares, which are sent to the base station(s). An overview of the communication scheme is depicted in Figure 1. From an overall system perspective, the nodes can be considered to cooperate to drive an initial state to the desired representative state $\overline{\mathbf{x}}$ by applying a control (which is the node's share) to the system via the state created by a previous node, and then relaying the updated state to the next node.

Since the controls drive the plant to $\overline{\mathbf{x}}$, the decryption algorithm is straight-forward as shown in Algorithm 2.

In order to decrypt, all the controls (or shares) and the entire plant/system must be known. Hence an attacker is forced to intercept all shares, or capture all nodes. Figure 3(a) illustrates how the initial state is driven to the desired representative state $\overline{\mathbf{x}}$. When a control is applied to a state, the 


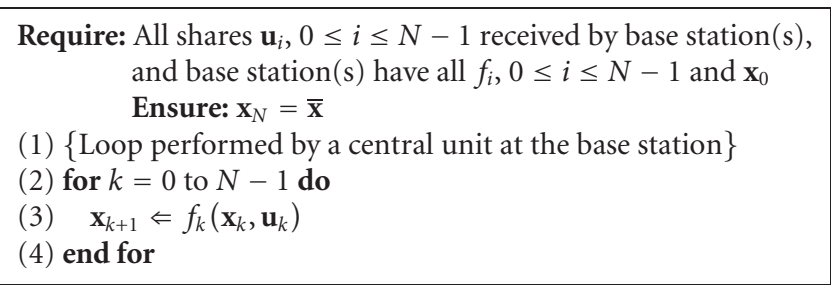

Algorithm 2: Decryption at base station(s) for VSN.

dynamical system is moved to a new state. Controls are applied successively to the dynamical system to drive the state to $\overline{\mathbf{x}}$ and hence reconstruct the representative image $I_{r}$.

Finally, each iteration in Algorithm 1 can be thought of as a round which adds confusion, hence Algorithm 1 mimics an iterated block cipher [31] with each round being performed by a single different node using a different key.

\subsection{Motivation for this paradigm}

There are many reasons why a dynamical systems approach is chosen for this problem. Such theory is well developed to handle external disturbances. For our problem, this is useful to ensure that image decryption is robust to natural (unintentional) system disturbances such as hardware noise, or intentional tampering. If we assume that the disturbance $\mathbf{w}_{i}$ is additive and constrained, for example bounded such that $\left\|\mathbf{w}_{i}\right\|<C$, then control laws can be designed such that the trajectory stays within some region around the desired representative state $\overline{\mathbf{x}}$ as illustrated in Figure $3(\mathrm{~b})$. If the ball around $\overline{\mathbf{x}}$ has radius $\rho_{0}$ or less, where $\rho_{0}$ is the variable accounting for perceptual similarity from Section 2.2, then decryption will still result in a good visual approximation of the desired image.

\subsection{Extensions}

This robustness allows for some additional advantages. The number of pair-wise keys that each sensor node carries to run the proposed algorithm is always 2 (i.e., $\mathcal{O}(1)$ ) regardless of the number of nodes in a cluster. This is because node $k$ only needs to receive the previous state from node $k-1$ and must send its current state to node $k+1$ requiring communication only among these nodes. This is a memory advantage because, in contrast to the sharing scheme presented in [25], the number of key fragments per node is $\mathcal{O}(\sqrt{N})$, where $N$ is the number of nodes. Finally, if each node is regarded as a vertex, and the communication between nodes is a directed edge, then the VSN is a directed graph. The number of fanouts, or outdegree of each node in our scheme is exactly 2 (one for transmitting to the next node, and one for transmitting to the base station(s)) regardless of the number of nodes in the cluster (i.e., $\mathcal{O}(1)$ ). However, the outdegree per node in [25] is again $\mathcal{O}(\sqrt{N})$. Also, the unidirectional nature of the internode communication in our paradigm promotes an optical sensor network architecture [32], which has been shown to be energy-efficient for communicating multimedia through free space [33].

\section{TANGRAM: ALGORITHM USING RANDOMNESS}

In this section we present an algorithm based on randomness (i.e., random vectors and random variables) and Lyapunov synthesis, which we call TANGRAM. ${ }^{8}$ In contrast to the algorithm presented by the authors in [27], the algorithm presented here is simpler lending itself more appropriately to distributed VSN security. Lyapunov synthesis provides a framework for generating the shares that drive an initial state to a desired state for nonlinear dynamical systems in general.

We first review Lyapunov stability theory. The equilibria of a discrete-time state space system in (3) are any solutions $\mathbf{x}_{\text {eq }}$ to (4):

$$
\mathbf{x}_{\mathrm{eq}}=f_{k}\left(\mathbf{x}_{\mathrm{eq}}, \mathbf{u}_{k}\right) .
$$

The goal is to design $\mathbf{u}_{k}$, such that starting from any initial state $\mathbf{x}_{0}$, the system converges to the unique equilibrium $\mathbf{x}_{\mathrm{eq}} \triangleq \overline{\mathbf{x}}$; when this is satisfied, $\overline{\mathbf{x}}$ is said to be globally asymptotically stable. A popular way to achieve this goal is via Lyapunov's stability theorem [34].

Theorem 3.1 (global asymptotic stability). The equilibrium $\mathbf{x}_{\mathrm{eq}}$ is globally asymptotically stable if there exists a function $V$ : $\mathbb{R}^{m \cdot n} \rightarrow \mathbb{R}$ such that

(1) $V\left(\mathbf{x}_{\mathrm{eq}}\right)=0$;

(2) there are continuous, strictly increasing functions $\alpha$ : $\mathbb{R} \rightarrow \mathbb{R}$ and $\beta: \mathbb{R} \rightarrow \mathbb{R}$, where $\alpha(0)=\beta(0)=0$, and

$$
\alpha\left(\left\|\mathbf{x}-\mathbf{x}_{\mathrm{eq}}\right\|\right) \leq V(\mathbf{x}) \leq \beta\left(\left\|\mathbf{x}-\mathbf{x}_{\mathrm{eq}}\right\|\right)
$$

for all $\mathbf{x} \in \mathbb{R}^{m \cdot n}$

(3) $V(\mathbf{x}) \rightarrow \infty$ as $\|\mathbf{x}\| \rightarrow \infty$;

(4) $V\left(\mathbf{x}_{k+1}\right)-V\left(\mathbf{x}_{k}\right)<0$ for all $k \geq 0$.

In Lyapunov synthesis, one begins by choosing the Lyapunov function $V$ that satisfies criteria 2 and 3 in Theorem 3.1. The goal is then to design $\mathbf{u}_{k}$ so that the equilibrium $\mathbf{x}_{\mathrm{eq}}$ is forced to be the desired $\overline{\mathbf{x}}$, which satisfies criterion 1 , and the overall system with the control incorporated satisfies criterion 4.

In [27] a linear system was proposed by the authors where the system matrix $A_{k}$ presented additional challenges as they had to be stored on-board the sensor nodes. Here we propose the following straightforward linear system given by (6):

$$
\mathbf{x}_{k+1}=\mathbf{x}_{k}+\mathbf{u}_{k} .
$$

Although there are many ways to design $\mathbf{u}_{k}=g_{k}\left(x_{k}, \overline{\mathbf{x}}\right)$, such that (6) is globally asymptotically stable with respect to the desired equilibrium $\overline{\mathbf{x}}$, our goal is that of secrecy/security, and hence it seems natural and practical to use a random approach.

Because we cannot expect to achieve global asymptotic stability in a purely random approach, we give Definition 3.2 to quantify the systems behavior at a particular time instance.

\footnotetext{
${ }^{8}$ The word "tangram" means a puzzle.
} 


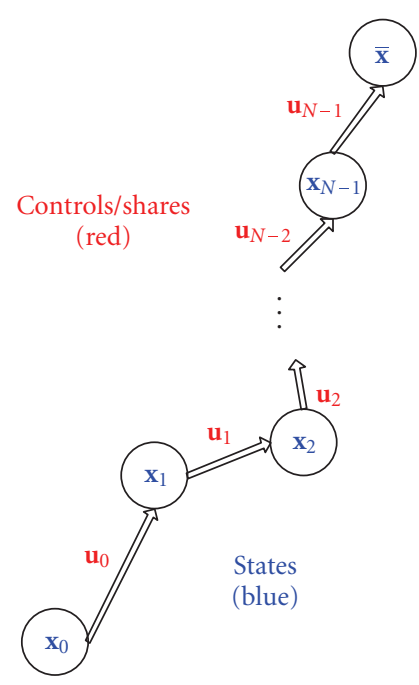

(a)

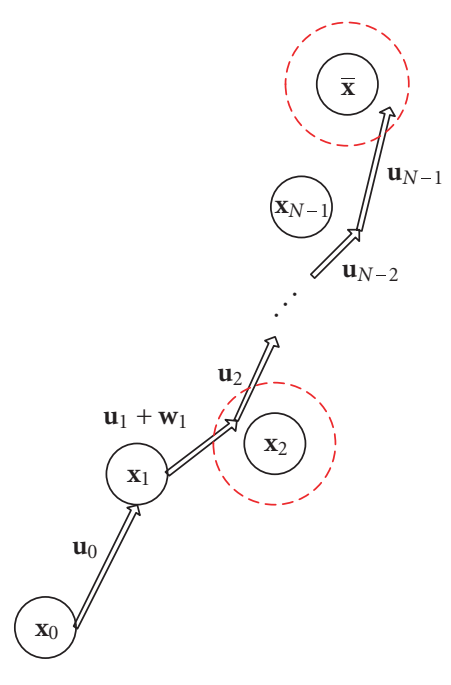

(b)

FIGURE 3: (a) Initial state being driven to the desired representative state $\overline{\mathbf{x}}$ by node controls; (b) noisy control/share causes offset in trajectory which stays within some ball.

Definition 3.2. We say a plant $\Sigma_{p}$ with equilibrium $\mathbf{x}_{\mathrm{eq}}$ is $b e$ having globally asymptotically stable at time $j$ if criteria 1 to 3 are satisfied in Theorem 3.1, and $V\left(\mathbf{x}_{k+1}\right)-V\left(\mathbf{x}_{k}\right)<0$ for all $k \leq j$.

Remarks 3.3. Definition 3.2 tells us that a system looks like a "promising" candidate for global asymptotic stability at a particular time instance. We now propose a random control law in Theorem 3.4 and state its property.

Theorem 3.4. Let $\Sigma_{p}$ be the plant whose state space equation is given by (6). Let

$$
\mathbf{u}_{k}=-\operatorname{sgn}\left(\mathbf{x}_{k}-\overline{\mathbf{x}}\right) \odot \mathbf{R}_{k}^{+},
$$

where $\odot$ denotes element-wise multiplication, sgn is the signum function operating on each element of the vector

$$
\operatorname{sgn}(y)= \begin{cases}1 & \text { if } y>0, \\ 0 & \text { if } y=0, \\ -1 & \text { if } y<0,\end{cases}
$$

and $\mathbf{R}_{k}^{+}$is a random vector taking on only nonnegative values and whose mean vector $E\left[\mathbf{R}_{k}^{+}\right]<\epsilon \cdot 2\left|\mathbf{x}_{k}-\overline{\mathbf{x}}\right|$ for $\epsilon>0$, where " $<$ " is taken element-by-element. Then $\Sigma_{p}$ has equilibrium $\overline{\mathbf{x}}$, and is behaving globally asymptotically stable at iteration $k$ (or node $k$, keeping in mind starting at 0 ) with probability greater than $(1-\epsilon)^{k+1}$.

Remarks 3.5. The theorem provides a lower bound to the probability that the system is behaving globally asymptotically stable at a given time. The simulation results will show that this lower bound is quite loose, meaning that decryption will result in images that fall in the similar region with much higher probability than this lower bound.
Proof. Without loss of generality, ${ }^{9}$ let us take the scalar case, in which we have

$$
x_{k+1}=x_{k}-\operatorname{sgn}\left(x_{k}-\bar{x}\right) R_{k}^{+} .
$$

First we can verify that $\bar{x}$ is indeed the equilibrium by substituting $\bar{x}$ into $x_{k}$ on the right-hand side. Next, let us define

$$
V(x)=(x-\bar{x})^{2}
$$

which indeed satisfies criteria 1 to 3 of Theorem 3.1. Starting at $k=0$, we see that

$$
\begin{gathered}
V\left(x_{1}\right)-V\left(x_{0}\right)=V\left(x_{0}-\operatorname{sgn}\left(x_{0}-\bar{x}\right) R_{0}^{+}\right)-V\left(x_{0}\right) \\
=\left(x_{0}-\operatorname{sgn}\left(x_{0}-\bar{x}\right) R_{0}^{+}-\bar{x}\right)^{2}-\left(x_{0}-\bar{x}\right)^{2}<0
\end{gathered}
$$

if $x_{0}-\operatorname{sgn}\left(x_{0}-\bar{x}\right) R_{0}^{+}$is closer to $\bar{x}$ than $x_{0}$ is; denote this event for $k=0$ as $E_{0}$. Then the probability of the complement is $P\left(\overline{E_{0}}\right)=\operatorname{Pr}\left\{R_{0}^{+} \geq 2\left|x_{0}-\bar{x}\right|\right\}$, which we can bound using Markov's inequality

$$
\operatorname{Pr}\left\{R_{0}^{+} \geq 2\left|x_{0}-\bar{x}\right|\right\} \leq \frac{E\left[R_{0}^{+}\right]}{2\left|x_{0}-\bar{x}\right|}<\epsilon,
$$

where the last inequality is due to $E\left[R_{k}^{+}\right]<\epsilon \cdot 2\left|x_{k}-\bar{x}\right|$. Therefore $P\left(E_{0}\right)>1-\epsilon$. Suppose that for some iteration $k-1, P\left(E_{0} \cap E_{1} \cap \cdots \cap E_{k-1}\right)>(1-\epsilon)^{k}$. Then we can show that $P\left(E_{k}\right)>(1-\epsilon)$ the same way we showed for $P\left(E_{0}\right)$. Since the $R_{k}^{+}$'s are independent for all $k, P\left(E_{0} \cap E_{1} \cap \cdots \cap E_{k}\right)>$ $(1-\epsilon)^{k+1}$, proving the theorem by induction.

\footnotetext{
9 TANGRAM operates pixel-by-pixel (or element-by-element), that is, only confusion is introduced, hence we can restrict our analysis to a single pixel/element.
} 
Remarks 3.6. To see why $P\left(\overline{E_{0}}\right)=\operatorname{Pr}\left\{R_{0}^{+} \geq 2\left|x_{0}-\bar{x}\right|\right\}$, we define two cases: $x_{0}<\bar{x}$, and $x_{0}>\bar{x}$. Noting that $R_{k}^{+}$is always nonnegative (the superscript + denotes this fact), if $x_{0}<\bar{x}$, then $-\operatorname{sgn}\left(x_{0}-\bar{x}\right)=1$, hence $-\operatorname{sgn}\left(x_{0}-\bar{x}\right) R_{0}^{+}$is positive; when this quantity is added to $x_{0}, x_{0}$ is increasing positively towards $\bar{x}$ in the correct direction. The event $\overline{E_{0}}$ occurs when this quantity added is two times the distance that $x_{0}$ is from $\bar{x}$, that is, $2\left|x_{0}-\bar{x}\right|$. The other case follows the same argument.

\subsection{Security analysis}

Next we analyze the security of this scheme. We begin with the notion of perfect secrecy. Given plaintext and ciphertext random variables $P$ and $C$, respectively, a cipher provides perfect secrecy if $I(P ; C)=0$, where $I(\cdot ; \cdot)$ denotes mutual information. Ciphers that incorporate a great deal of randomness, such as the one-time pad are good candidates for perfect secrecy. To show that TANGRAM satisfies perfect secrecy under certain conditions, we present Lemma 3.7, which is based on TANGRAM parameters. Then we discuss how Lemma 3.7 is related to TANGRAM.

Lemma 3.7. Let $U=\sigma \cdot R^{+}$where $R^{+}$is a positive continuous random variable whose mean is $E[U]=\theta=\left|\theta_{1}-\theta_{2}\right|$, where $\theta_{1}, \theta_{2}$ are positive continuous random variables, $\sigma=\operatorname{sgn}\left(\theta_{1}-\right.$ $\left.\theta_{2}\right)$. If $h(\theta)=h\left(\theta_{1}\right)$, then $I\left(U ; \theta_{2}\right)=0$.

Proof. First we write $h\left(U, \theta \mid \theta_{2}, \sigma\right)$ in two ways using the chain rule

$$
\begin{aligned}
h\left(U, \theta \mid \theta_{2}, \sigma\right) & =h\left(U \mid \theta_{2}, \sigma\right)+h\left(\theta \mid U, \theta_{2}, \sigma\right) \\
& =h\left(\theta \mid \theta_{2}, \sigma\right)+h\left(U \mid \theta, \theta_{2}, \sigma\right) .
\end{aligned}
$$

Next we can simplify all the quantities. Throughout we use the fact that $\theta$ can be written as $\theta=\left|\theta_{1}-\theta_{2}\right|=\operatorname{sgn}\left(\theta_{1}-\theta_{2}\right)$. $\left(\theta_{1}-\theta_{2}\right)=\sigma \cdot\left(\theta_{1}-\theta_{2}\right)$ :

$$
\begin{aligned}
h\left(U \mid \theta_{2}, \sigma\right) & =h\left(\sigma \cdot R^{+} \mid \theta_{2}, \sigma\right)=h\left(R^{+} \mid \theta_{2}\right), \\
h\left(\theta \mid U, \theta_{2}, \sigma\right) & =h\left(\sigma \cdot\left(\theta_{1}-\theta_{2}\right) \mid \sigma \cdot R^{+}, \theta_{2}, \sigma\right) \\
& =h\left(\theta_{1} \mid R^{+}\right)=h\left(\theta \mid R^{+}\right), \\
h\left(\theta \mid \theta_{2}, \sigma\right) & =h\left(\sigma \cdot\left(\theta_{1}-\theta_{2}\right) \mid \theta_{2}, \sigma\right) \\
& =h\left(\theta_{1}\right)=h(\theta), \\
h\left(U \mid \theta, \theta_{2}, \sigma\right) & =h\left(\sigma \cdot R^{+} \mid \theta, \theta_{2}, \sigma\right) \\
& =h\left(\sigma \cdot R^{+} \mid \theta, \sigma\right)=h\left(R^{+} \mid \theta\right) .
\end{aligned}
$$

We have used the fact that $h(\theta)=h\left(\theta_{1}\right)$ in the last equalities of (15). For the second equality in (16), we used the fact that $\theta$ is the true mean of $R^{+}$, hence $\theta_{2}$ provides no additional information since $\theta=\left|\theta_{1}-\theta_{2}\right|$. Now substituting (14)-(16) into (13), we get

$$
\begin{aligned}
h\left(R^{+} \mid \theta_{2}\right)+h\left(\theta \mid R^{+}\right) & =h(\theta)+h\left(R^{+} \mid \theta\right), \\
h\left(R^{+} \mid \theta_{2}\right) & =h\left(\theta, R^{+}\right)-h\left(\theta \mid R^{+}\right) \\
& =h\left(R^{+}\right) .
\end{aligned}
$$

Therefore $I\left(R^{+} ; \theta_{2}\right)=h\left(R^{+}\right)-h\left(R^{+} \mid \theta_{2}\right)=0$.
Let us apply Lemma 3.7 to TANGRAM on a pixel-bypixel or element-by-element basis. We only assume that the attacker has intercepted one share $u_{i}$ for some $i$, and that none of the states $x_{k}$ nor the mean are known. Let $\theta_{1}=x_{i}$ and $\theta_{2}=\bar{x}$, and also multiply the mean by $2 \epsilon$. If the entropy of $x_{i}$ is equal to the entropy of the mean, then perfect secrecy of a single pixel is achieved.

We note that the attacker does not have access to the mean, and we can further enhance the security by loading each sensor node with different probability density functions (PDFs) for generating $R_{k}^{+}$and not revealing them; the PDFs are a parameter of the algorithm, which can be considered a node-dependent key. If the aforementioned entropies are not equal, we give a more general but weaker result in Lemma 3.8.

Lemma 3.8. Let $U=\sigma \cdot R^{+}$where $R^{+}$is a positive continuous random variable whose mean is $E[U]=\theta=\left|\theta_{1}-\theta_{2}\right|$, where $\theta_{1}, \theta_{2}$ are positive continuous random variables, $\sigma=\operatorname{sgn}\left(\theta_{1}-\right.$ $\left.\theta_{2}\right)$. Then

$$
I\left(\theta_{2} ; U\right)=h\left(U \mid \theta_{1}\right)-h(U \mid \theta) .
$$

Proof. This time we write $h\left(U, \theta \mid \theta_{1}, \sigma\right)$ in two ways using the chain rule

$$
\begin{aligned}
h\left(U, \theta \mid \theta_{1}, \sigma\right) & =h\left(U \mid \theta_{1}, \sigma\right)+h\left(\theta \mid U, \theta_{1}, \sigma\right) \\
& =h\left(\theta \mid \theta_{1}, \sigma\right)+h\left(U \mid \theta, \theta_{1}, \sigma\right), \\
h\left(U \mid \theta_{1}, \sigma\right) & =h\left(\sigma \cdot R^{+} \mid \theta_{1}, \sigma\right)=h\left(R^{+} \mid \theta_{1}\right), \\
h\left(\theta \mid U, \theta_{1}, \sigma\right) & =h\left(\sigma \cdot\left(\theta_{1}-\theta_{2}\right) \mid \sigma \cdot R^{+}, \theta_{1}, \sigma\right) \\
& =h\left(\theta_{2} \mid R^{+}\right), \\
h\left(\theta \mid \theta_{1}, \sigma\right) & =h\left(\sigma \cdot\left(\theta_{1}-\theta_{2}\right) \mid \theta_{1}, \sigma\right)=h\left(\theta_{2}\right), \\
h\left(U \mid \theta, \theta_{1}, \sigma\right) & =h\left(\sigma \cdot R^{+} \mid \theta, \theta_{1}, \sigma\right) \\
& =h\left(\sigma \cdot R^{+} \mid \theta, \sigma\right)=h\left(R^{+} \mid \theta\right) .
\end{aligned}
$$

Combining the constituents

$$
\begin{aligned}
h\left(R^{+} \mid \theta_{1}\right)+h\left(\theta_{2} \mid R^{+}\right) & =h\left(\theta_{2}\right)+h\left(R^{+} \mid \theta\right), \\
h\left(R^{+} \mid \theta_{1}\right)-h\left(R^{+} \mid \theta\right) & =h\left(\theta_{2}\right)-h\left(\theta_{2} \mid R^{+}\right) \\
& =I\left(\theta_{2} ; R^{+}\right) .
\end{aligned}
$$

Having provided analysis for the simplest case of a single interception, we give an analogy of taking a number $\tau$ and randomly breaking it into $N$ numbers $\tau_{0}, \tau_{1}, \ldots, \tau_{N-1}$ such that the sum of these $N$ numbers is equal to $\tau$. If we give one or two of these $\tau_{i}$ to someone and ask them to guess the original number $\tau$, it would be as difficult as deducing an entire puzzle from one or two pieces alone. ${ }^{10}$

\footnotetext{
${ }^{10}$ We note that our analogy partitions an image spatially, whereas in TANGRAM, partitioning is performed at the pixel level. This is because spatial partitions may still reveal some semantic content of the image in question.
} 


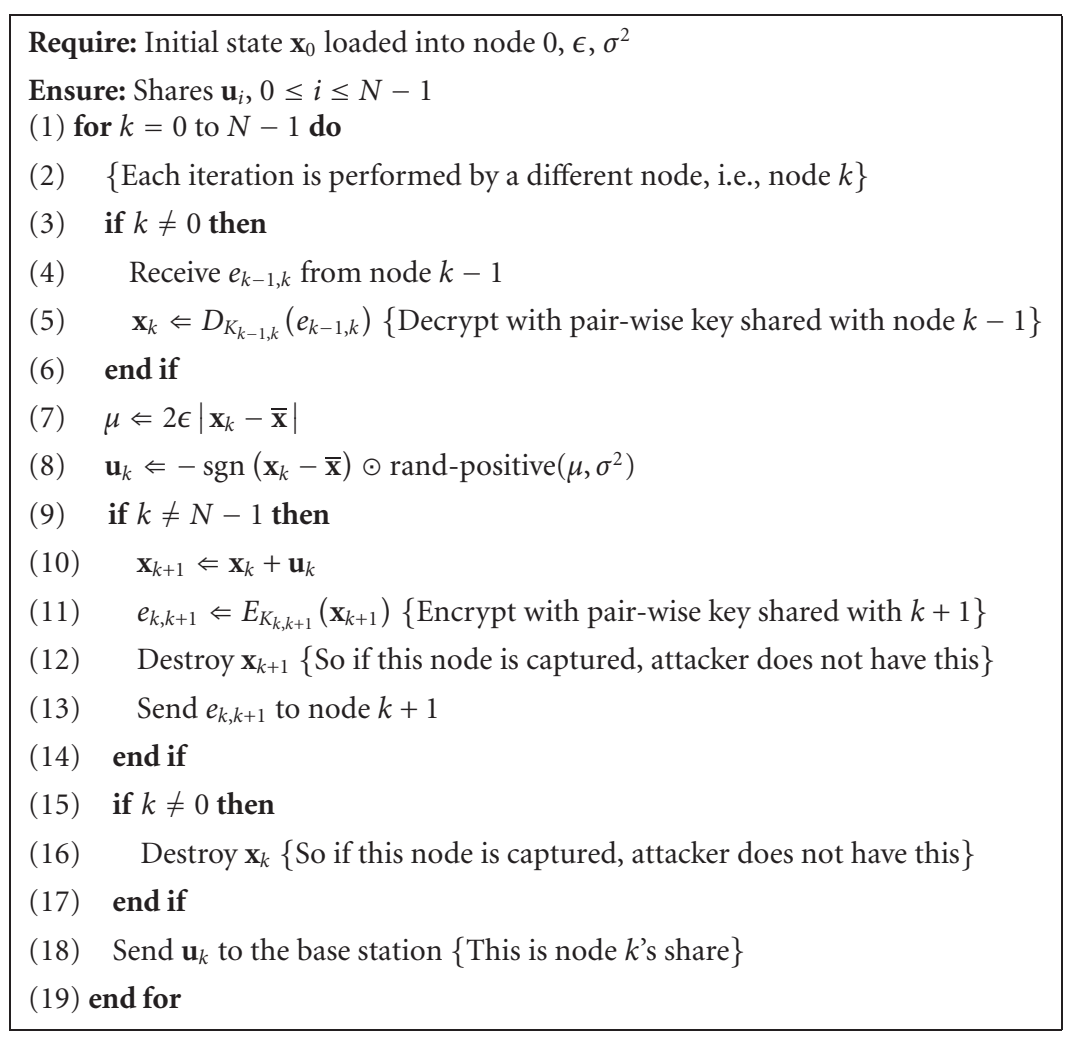

Algorithm 3: TANGRAM.

Let us consider the scalar case again. For the case of more than one interception, assume that $t$ shares (where this $t$ is from the goals in Section 2.1) are intercepted. Then we want to ensure that decryption using these $t$ nodes falls in the dissimilar region with high probability. Since the shares are generated randomly, suppose that the first $t$ shares are intercepted. Then this $t$ should satisfy $(21)$ for $\delta>0$ :

$$
\operatorname{Pr}\left\{\left|\bar{x}-x_{0}-\sum_{k=0}^{t-1} u_{k}\right|>\rho_{1}\right\}>1-\delta .
$$

As we will see in Section 3.3, this criterion is coupled with robustness constraints, which renders the closed-form derivation of $t$ intractable. Hence the determination of $t$ will be left to the devices of simulation found in Section 4.3.

\subsection{Implementation}

The particulars of the TANGRAM algorithm are summarized in Algorithm 3 for ease of reference. We now examine the implementation of the TANGRAM algorithm and show that it is indeed cost efficient, robust, and suited for VSNs.

How efficient is Algorithm 3 in terms of share size? This question is inherently linked to the issue of compression. If we look at this question at the pixel level, then the cost of one pixel is its absolute value; hence the cost of all shares is $\left|u_{0}\right|+\left|u_{1}\right|+\cdots+\left|u_{N-1}\right|$. Intuitively, a pixel with smaller absolute value will require fewer bits to encode than a pixel with larger absolute value, and hence from this point of view, minimizing this cost achieves a crude form of compression.
Definition 3.9 (Optimal share size). The shares $\mathbf{u}_{0}, \mathbf{u}_{1}, \ldots$, $\mathbf{u}_{N-1}$ generated by Algorithm 3 achieve optimal share size if

$$
\left|\mathbf{u}_{0}\right|+\left|\mathbf{u}_{1}\right|+\cdots+\left|\mathbf{u}_{N-1}\right| \leq\left|\overline{\mathbf{x}}-\mathbf{x}_{0}\right|
$$

where $|\cdot|$ is the element-wise absolute value.

Definition 3.9 is motivated by the fact that if the shares overshoot the desired representative image, and oscillate about the representative image, then they will effectively have total absolute size greater than if the shares never overshoot. Theorem 3.4 and its proof provides us with a result on optimal share size as stated in Corollary 3.10.

Corollary 3.10. The shares produced by Algorithm 3 achieve optimal share size with probability greater than

$$
(1-2 \epsilon)^{N} \text {. }
$$

Remarks 3.11. The factor 2 comes from the fact that in order to achieve optimal share size, we require $R_{k}^{+}<\left|x_{k}-\bar{x}\right|$ for all $k$; see the proof for Theorem 3.4.

\subsection{Robustness}

In this section we assume that noise (either through unintentional sensor errors, miscalibrations, or intentional tampering) is added to the shares. If we use Algorithm 3, we can write decryption as $\mathbf{x}_{0}+\sum_{k=0}^{N-1}\left(\mathbf{u}_{k}+\mathbf{w}_{k}\right)=\left(\mathbf{x}_{0}+\sum_{k=0}^{N-1} \mathbf{u}_{k}\right)+$ $\sum_{k=0}^{N-1} \mathbf{w}_{k}=\overline{\mathbf{x}}+\sum_{k=0}^{N-1} \mathbf{w}_{k}$, where we have also assumed that imperfect decryption (i.e., all the shares and the initial state 
do not add up to be the representative state) is incorporated into the noise vectors $\mathbf{w}_{k}$. From Section 2.2, if $\left\|\sum_{k=0}^{N-1} \mathbf{w}_{k}\right\|<$ $\rho_{0}$ for the perceptual similarity constant $\rho_{0}$, then decryption will still reveal the semantics of $\overline{\mathbf{x}}$. This constraint may be unreasonable under an intentional attack situation, however our assumptions in Section 2.1.2 restrict the number of shares attacked to no more than $t$, thus restricting the effect on the decrypted image.

We exercise this assumption and assume the worst case scenario, in which none of the $t$ tampered shares may be used. Let $S$ be the set of shares of cardinality $t$ that are ruined. Then it is natural to use the complement $\bar{S}$, resulting in $\mathbf{x}_{0}+\sum_{k=0}^{N-1} I_{\bar{S}}\left(\mathbf{u}_{k}\right) \mathbf{u}_{k}$, where

$$
I_{A}(x)= \begin{cases}1 & \text { if } x \in A \\ 0 & \text { if } x \notin A\end{cases}
$$

is the indicator function. If $\left\|\overline{\mathbf{x}}-\left(\mathbf{x}_{0}+\sum_{k=0}^{N-1} I_{\bar{S}}\left(\mathbf{u}_{k}\right) \mathbf{u}_{k}\right)\right\|<\rho_{0}$, then decryption will reveal the semantics as desired. There are two questions that need to be answered. First, what is the maximum $t=|S|$ for which a perceptually acceptable reconstruction is possible? Second, how does the base station(s) determine the set $S$ ?

To address the first question, let us consider the scalar case. Without loss of generality, assume the last $t$ shares are ruined while the first $N-t$ shares are pristine. Since the shares are generated randomly, given a $\delta>0$, maximize $t$ such that

$$
\operatorname{Pr}\left\{\left|\bar{x}-x_{0}-\sum_{k=0}^{N-t-1} u_{k}\right|<\rho_{0}\right\}>1-\delta .
$$

This constraint probability can be written as

$$
\begin{aligned}
& \operatorname{Pr}\left\{-\rho_{0}<\bar{x}-x_{0}-\sum_{k=0}^{N-t-1} u_{k}<\rho_{0}\right\} \\
& =\operatorname{Pr}\left\{\bar{x}-x_{0}-\rho_{0}<\sum_{k=0}^{N-t-1} u_{k}<\bar{x}-x_{0}+\rho_{0}\right\} .
\end{aligned}
$$

If we let $Y=U_{1}+U_{2}+\cdots, U_{N-t-1}$ be the random variable accounting for the sum of the random shares, and $f_{Y}(y)$ its PDF, then the problem for the scalar case is to maximize $t$ such that

$$
\int_{\bar{x}-x_{0}-\rho_{0}}^{\bar{x}-x_{0}+\rho_{0}} f_{Y}(y) d y>1-\delta
$$

Even for the scalar case, this problem is formidable, since the PDFs of the random variables $U_{i}$ have unknown variable means, and hence is best suited for computational simulations. $^{11}$

${ }^{11}$ In addition to the constraint given by (27), (21) should also be satisfied for security reasons. But obviously this makes the problem even more difficult.
The second problem can be rephrased as finding the set $\bar{S}$ such that $\mathbf{x}_{0}+\sum_{k=0}^{N-1} I_{\bar{S}}\left(\mathbf{u}_{k}\right) \mathbf{u}_{k}$ is closest to $\overline{\mathbf{x}}$. Without any side information, this problem is nontrivial. In fact, this problem in general (without side information) is just as hard as the knapsack problem known to be NP-complete [35]. To make this problem tractable, the usual device is to embed an authentication code (side information), such that the base station(s) can verify whether each share is pristine or corrupted. In this way, the base station(s) can construct the desired set $\bar{S}$.

\section{SIMULATION AND INTERPRETATION}

In this section we present the simulation results and discuss their meaning. We present results from two images. The first image used is shown in Figure 4 and has dimensionality of $587 \times 393$ while the second image has dimensionality of $512 \times 512$ and can be seen in Figure 12. The significance of choosing different image dimensions in our work is to demonstrate that although the perceptual constants are typically determined empirically for each image, they may also be reused for images of approximately the same dimensions. This property is desirable for sensor networks which are often required to operate as autonomously as possible without having to readjust its parameters.

\subsection{Choosing $\rho_{0}$ and $\rho_{1}$}

As one of the first steps in our implementation, we must estimate values of the similarity and dissimilarity constants $\rho_{0}$ and $\rho_{1}$ respectively as discussed in Section 2.2. By adding zero-mean white Gaussian noise with different variances, and visually inspecting the outcomes, we find that with a variance of no more than 50, the image is still understandable, while with a variance of at least 500, the image is incomprehensible. To determine the norm, we ran several experiments with variances 50 and 500, and computed the average norm of the noise, which turns out to be $\rho_{0}=24000$ and $\rho_{1}=37000$, respectively.

\subsection{Random distribution}

TANGRAM is based on a positive continuous distribution. In our simulations, we use the lognormal distribution, since the mean and variance can be controlled independently. ${ }^{12}$ The lognormal PDF is given in (28), and its mean $\mu$ and variance $\sigma^{2}$ are given in (29), respectively:

$$
\begin{aligned}
f(x) & =\frac{1}{s \sqrt{2 \pi}} \frac{1}{x} e^{-(\ln (x)-m)^{2} / 2 s^{2}}, \\
\mu & =e^{m+s^{2} / 2}, \\
\sigma^{2} & =e^{2\left(m+s^{2}\right)}-e^{2 m+s^{2}} .
\end{aligned}
$$

\footnotetext{
12 Other one-sided continuous distributions such as exponential, chisquared have PDFs based on one parameter which controls both the mean and variance in tandem.
} 


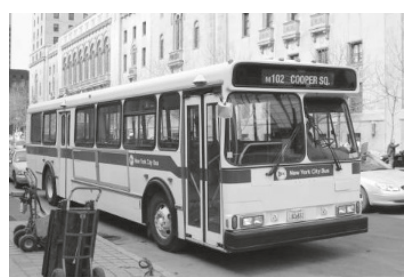

(a)

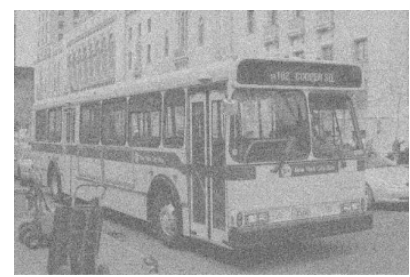

(b)

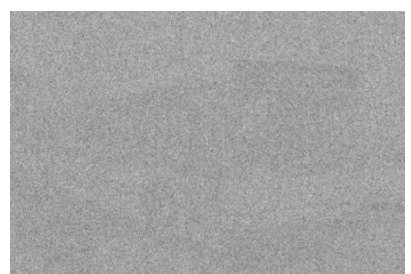

(c)

FIgUre 4: (a) Original bus (๔ come.to/torontobus); (b) bus with AWGN $\sigma^{2}=50$; (c) bus with AWGN $\sigma^{2}=500$.

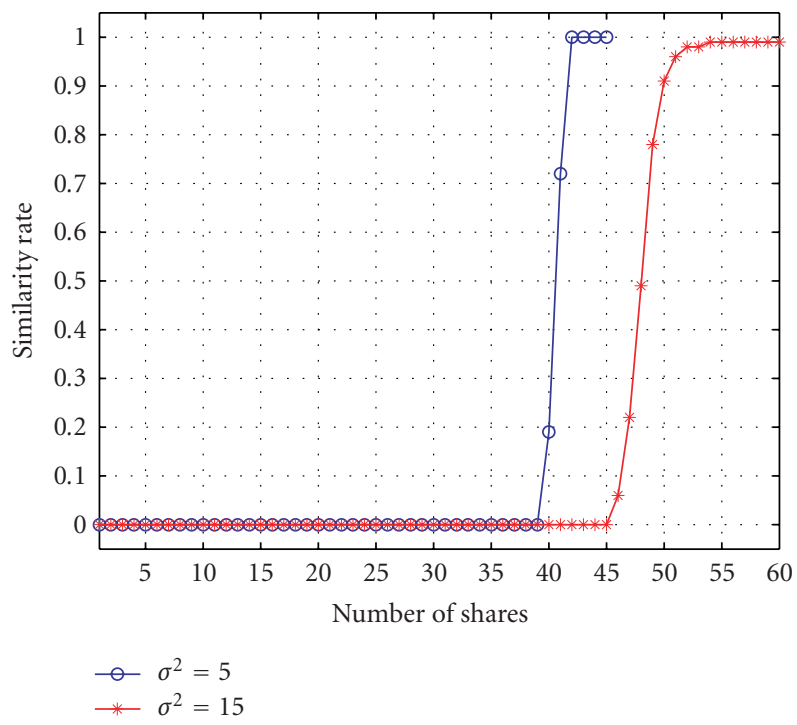

(a)

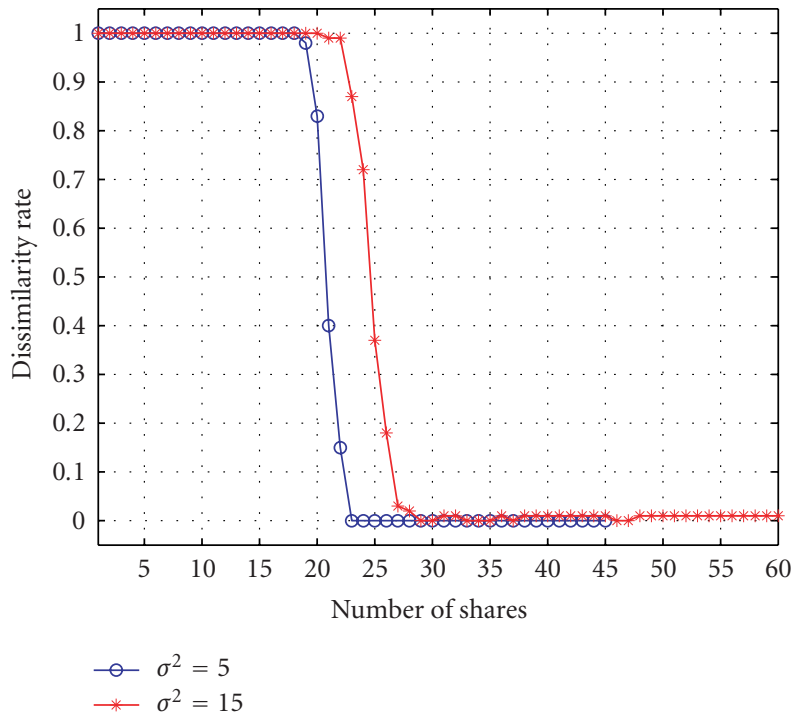

(b)

FIGURE 5: (a) Similarity rate; (b) dissimilarity rate.

The mean is dependent on the parameter $\epsilon$ (see Algorithm 3 ). In our simulations we use two values $\epsilon=10^{-2}$ and $10^{-3}$. The variance can be defined by the user. In our simulations, we use two values for the variance, $\sigma^{2}=5$ and 15 . We will discuss the implications of $\epsilon$ and $\sigma^{2}$ in Section 4.5.

\subsection{Determining suitable $N$ and $t$}

Next we want to determine how many sensor nodes are needed so that decryption is satisfactory. Figure $5(a)$ shows the rate (or simulated probability) that decryption will result in an image that falls in the similar region characterized by $\rho_{0}$. We see that at least 40 shares are necessary before a decrypted image falls in the similar region with high probability.

The value of $t$, that is, the total number of interceptions allowed, can be stated as the number of shares an attacker can intercept before decryption (using this number of shares) results in an image that no longer falls in the dissimilar region (i.e., it either falls in the fuzzy or similar region). Figure 5(b) shows the rate (or simulated probability) that decryption will result in an image that falls in the dissimilar region. We see that 20 shares or less will result in an image that falls in the dissimilar region with high probability. Since the determination of $\rho_{1}$ was empirical, we choose a conservative $t$, which is half of 20 , giving $t=10$.

Since we require at least 40 nodes for decryption, but allow 10 nodes to be intercepted, we choose $N=40+10=50$ as the number of nodes in a cluster. Figure 6 shows an example of a share, decryption using 10 shares, and decryption using 40 shares. Images have been scaled appropriately for highest perceptual quality.

\subsection{Convergence and security}

In Section 4.3 we presented the simulated probability of decryption falling in the similar and dissimilar regions depending on the number of shares. An important question to ask is the following: how does decryption transition between the similar, fuzzy, and dismilar regions as the number of shares available is varied? This question not only addresses convergence, but also security from the point of view that if an attacker is able to intercept one extra share, how does this additional interception improve his ability to comprehend the decrypted image. 


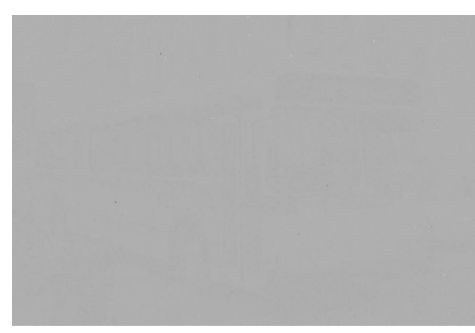

(a)

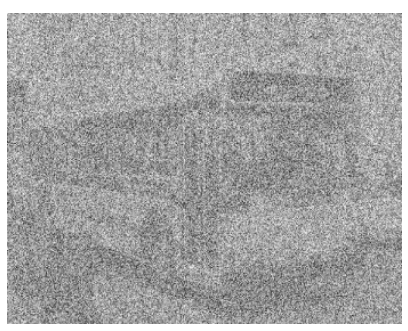

(b)

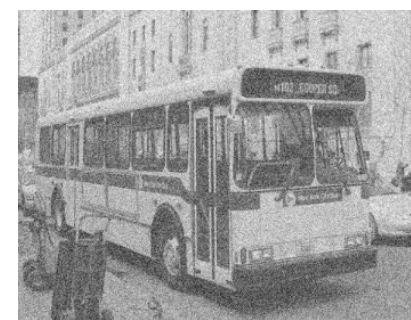

(c)

Figure 6: (a) Sample share; (b) decryption using $t=10$ shares; (c) decryption using 40 shares.

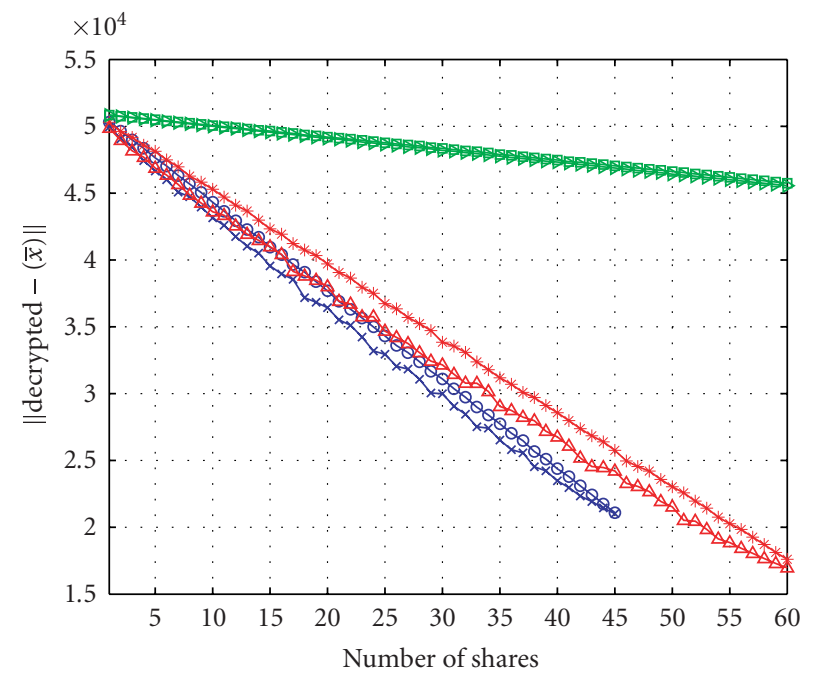

$\rightarrow$ Average $\left(\sigma^{2}=5, \epsilon=10^{-2}\right) \quad * \quad \operatorname{Minimum}\left(\sigma^{2}=5, \epsilon=10^{-2}\right)$

$\because$ Average $\left(\sigma^{2}=15, \epsilon=10^{-2}\right) \triangle$ Minimum $\left(\sigma^{2}=15, \epsilon=10^{-2}\right)$

$\rightarrow$ Average $\left(\sigma^{2}=5, \epsilon=10^{-3}\right) \rightarrow \operatorname{Minimum}\left(\sigma^{2}=5, \epsilon=10^{-3}\right)$

(a)

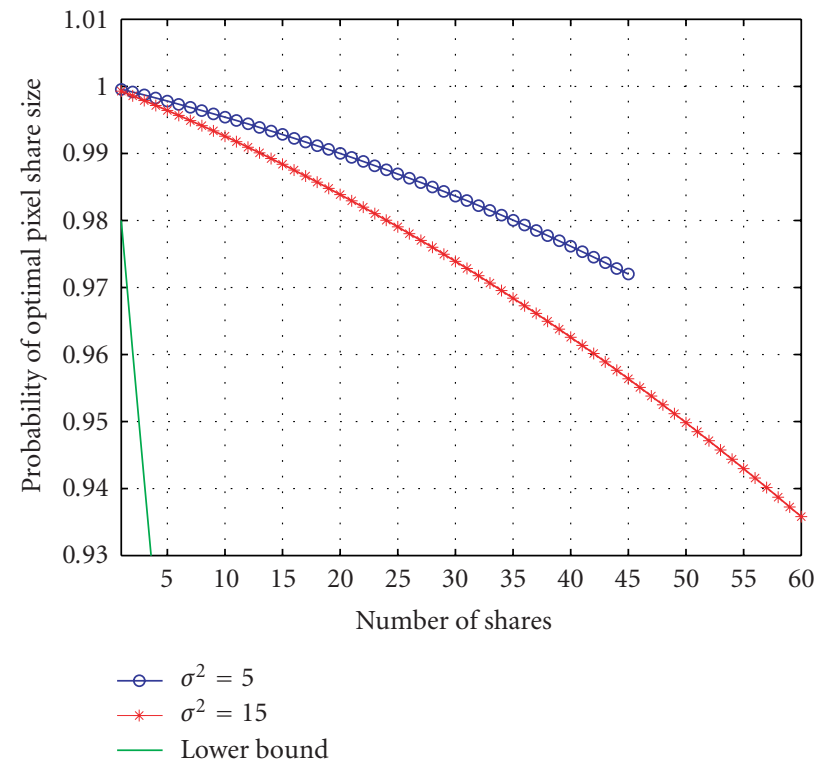

(b)

Figure 7: (a) $\left\|\mathbf{x}_{0}+\sum_{k=0}^{n} \mathbf{u}_{k}-\overline{\mathbf{x}}\right\|$ as a function of the number of shares $n$; (b) $\operatorname{Pr}\left\{\sum_{k=0}^{n}\left|u_{k}\right| \leq\left|\bar{x}-x_{0}\right|\right\}$ as a function of the number of shares $n$.

Figure 7(a) shows the average and minimum distances between the decrypted image and the representative image. We see that with more shares, the distance becomes closer, that is, decryption results in a better visual approximation of the representative image. Furthermore, we see that this phenomenon happens linearly. From a security point of view, each share intercepted linearly improves the attackers ability to comprehend the secret image. However, as long as the number of shares intercepted by an attacker does not exceed $t$, decryption will fall in the dissimilar region. In terms of robustness, each share that is lost or damaged will degrade the decrypted image linearly. Again if no more than $t$ shares are lost or damaged, decryption will not suffer provided that these $t$ shares are not used in decryption when they are damaged.

Finally, Figure 7(b) shows the probability that the pixels in a collection of shares have optimal share size as defined in Definition 3.9. We see that the lower bound provided by
Corollary 3.10 is rather modest, and in fact pixels are likely to achieve optimal share size with high probability.

\subsection{Effect of $\epsilon$ and $\sigma^{2}$}

In the plots above, we have shown the results for varying $\epsilon$ and $\sigma^{2}$. From Algorithm 3, we know that the mean of the distribution is a function of $\epsilon$. The smaller we make $\epsilon$, the smaller the mean is. From Figure 7(a), we see that when $\epsilon=10^{-3}$, the decrypted image is far from the representative image. Since this $\epsilon=10^{-3}$ is smaller than $\epsilon=10^{-2}$, the mean is smaller, and hence each share size is smaller, and it takes many more shares to result in a good visual approximation.

Similarly, when the variance is increased, we see that the simulation with the larger $\sigma^{2}=15$ also converges slower than $\sigma^{2}=5$. This is demonstrated in Figure 5(a), which shows that slightly more shares are required for decryption 


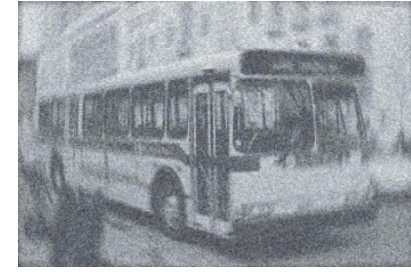

(a)

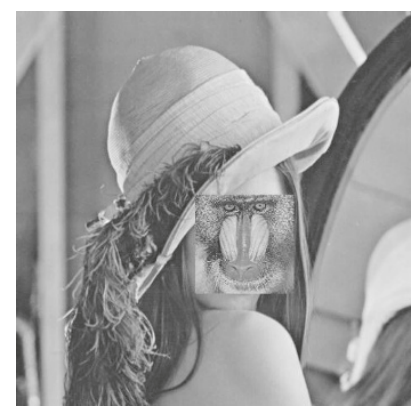

(b)

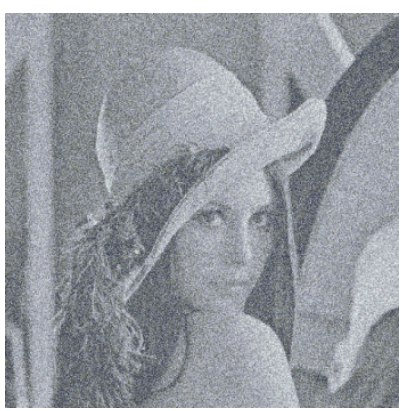

(c)

Figure 8: (a) Unintentional tampering: decrypted result of unregistered shares; (b) intentional tampering: Lena masked; (c) decryption using 40 shares resists tampering and discloses Lena's face.

to land in the similar region for the $\sigma^{2}=15$ case. Of course at the same time, we can allow attackers to intercept more shares before leaving the dissimilar region when $\sigma^{2}$ is larger as shown in Figure 5(b).

\subsection{Tampering}

In this section we briefly examine the effects of unintentional and intentional tampering. Figure 8 (a) is the visually acceptable result of combining 40 shares that are not registered; that is, the 40 nodes each have different representative images that are random rotations of one another over a uniform distribution of -2.5 degrees to 2.5 degrees. Such misalignments may be caused by misaligned cameras for example, and hence we classify them as unintentional tampering. Figure 8(b) shows Lena's face being intentionally masked by a mandrill's face. Five nodes were given this tampered representative image, and the result of decrypting with 40 shares

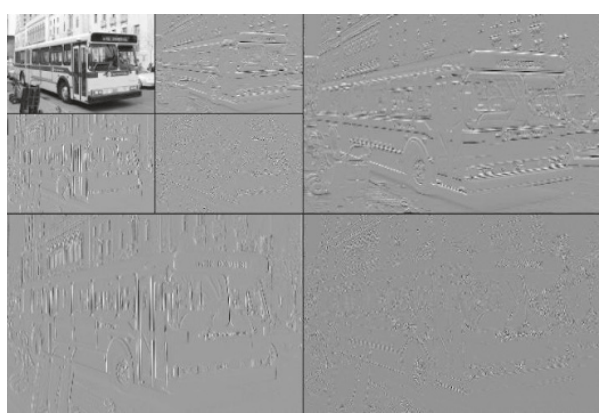

(a)

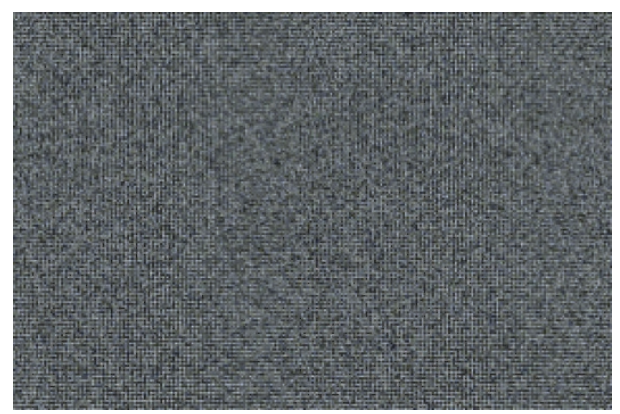

(b)

FIGURE 9: (a) 2-level Haar wavelet decomposition; (b) a share created from the Haar wavelet domain.

is shown in Figure 8(c). Intuitively, since the majority of the shares are unaffected, this majority visually overwhelms the tampered minority. However this resilience against tampering comes at the cost of redundancy in the network, as a large majority is needed. This agrees with Sections 4.3 and 4.4 in that $N$ is always much larger than $t$, implying only a small number of shares can be compromised compared to the total number of shares in the network, thus completing our insight into the tradeoff between resilience and redundancy.

Up to this point, we have considered sharing the pixels of an image. Image compression usually takes place in a domain other than the pixel domain, that is, a frequency domain [30]. In this section we use a 2-level Haar wavelet decomposition, which can be seen in Figure 9(a). In addition, we exercise rudimentary compression by discarding the diagonal high frequency subbands in both levels (i.e., the lower right corners of both levels in Figure 9(a)) to demonstrate the feasibility of extending TANGRAM to incorporate more standard compression techniques. Each node first applies a 2-level Haar wavelet decomposition to its representative image, and then TANGRAM proceeds exactly as outlined in Algorithm 3 on the wavelet subbands with the exception that the diagonal high frequency subbands in both levels are discarded. At the base station(s), the discarded subbands are replaced by zeros, and then Algorithm 2 is applied on all subbands. Finally the inverse wavelet transform is performed, resulting in a good visual approximation as shown 


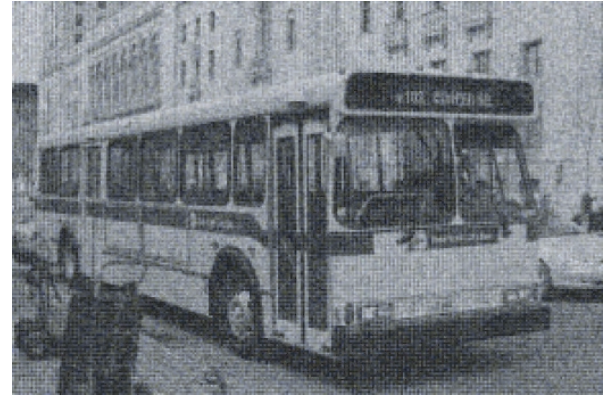

(a)

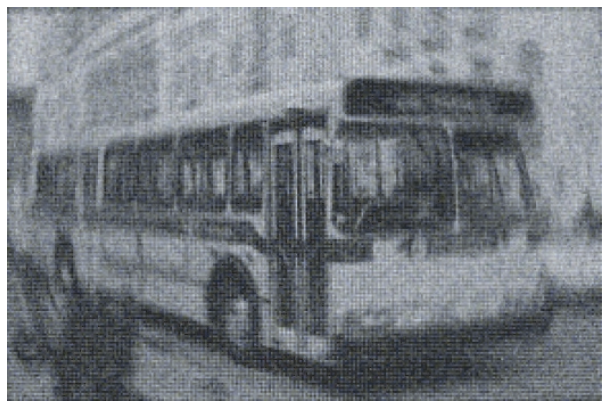

(b)

Figure 10: (a) Decryption of Haar wavelet compressed shares; (b) decryption of unregistered Haar wavelet compressed shares.

in Figure 10(a). We will refer to this extension as waveletTANGRAM.

Next we compare wavelet-TANGRAM to TANGRAM for a few special attacks to demonstrate the feasibility of extending TANGRAM. If an attacker arbitrarily intercepts one wavelet-TANGRAM share and performs the appropriate inverse wavelet transform, then the resulting image is unintelligible as shown in Figure 9(b); this is expected and analogous to Figure 6(a). If the representative images are misaligned as described in Section 4.6, decryption with 40 shares will still result in a good visual approximation as shown in Figure 10(b).

\section{CONCLUSIONS}

This paper provides a paradigm for distributing privacy and confidentiality in a visual sensor network. We have presented a simple algorithm, TANGRAM, which meets low complexity requirements of VSNs, hence allowing for other applications to coexist on-board each sensor. In addition, we have provided simple metrics for measuring perceptual similarity, robustness, security, and the optimality of share sizes. We have provided a comprehensive simulation and discussion of the results encompassing significant aspects of the problem. Future work will look at combining the proposed algorithm within an image/video compression algorithm compatible with VSNs as well as developing general design insights for

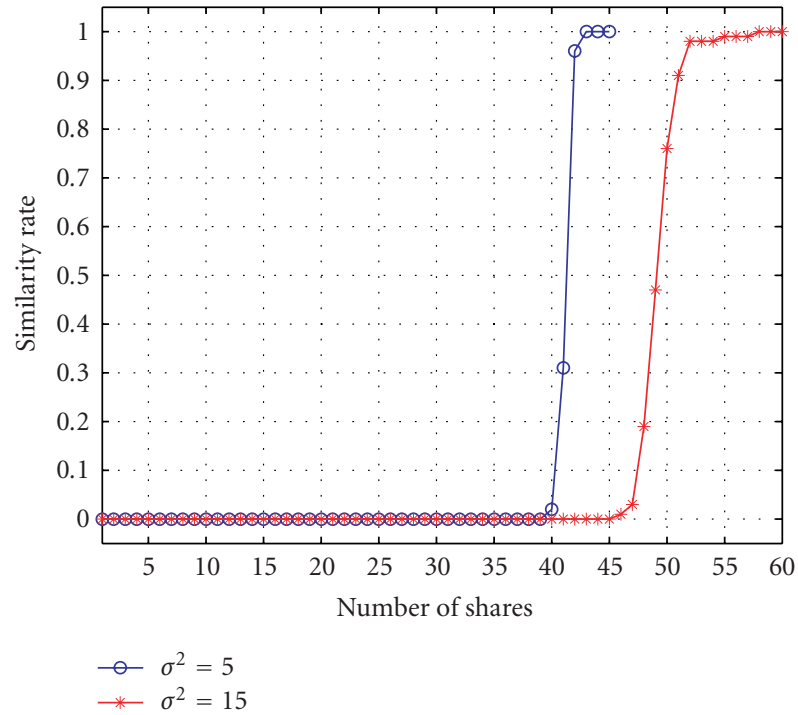

(a)

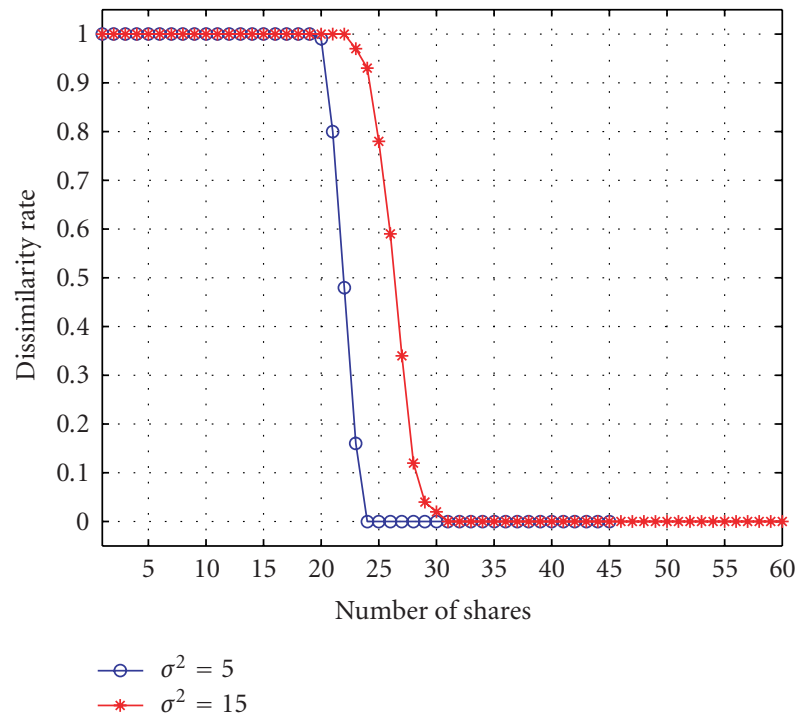

(b)

FIgURE 11: (a) Similarity rate; (b) dissimilarity rate.

the generation of secure shares in deterministic and random cases.

\section{APPENDIX}

\section{A. ADDITIONAL SIMULATION RESULTS}

Although the perceptual constants $\rho_{0}$ and $\rho_{1}$ were generated empirically for the bus image, we show in this section that highly similar results are achieved for a different image of similar dimensions using these constants. This demonstrates that we can choose $N$ and $t$ ahead of time if the image 


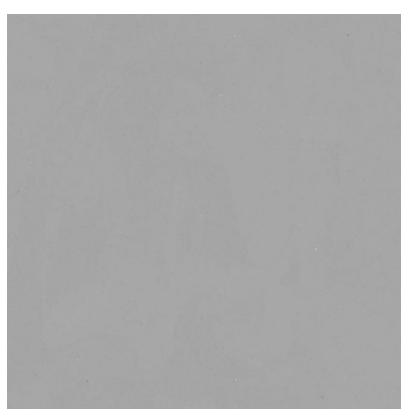

(a)

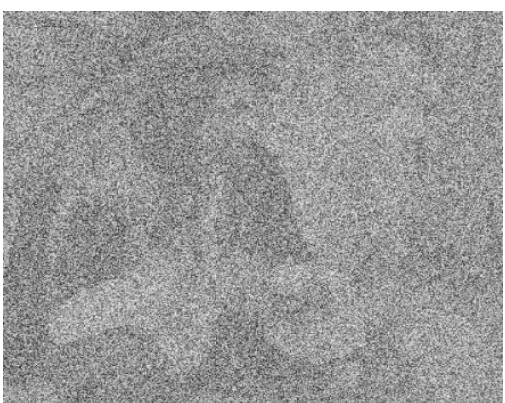

(b)

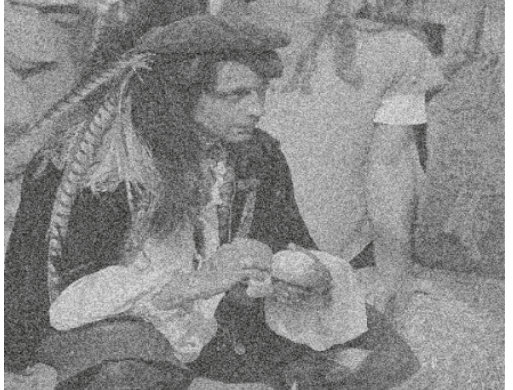

(c)

Figure 12: (a) Sample share; (b) decryption using $t=10$ shares; (c) decryption using 40 shares.

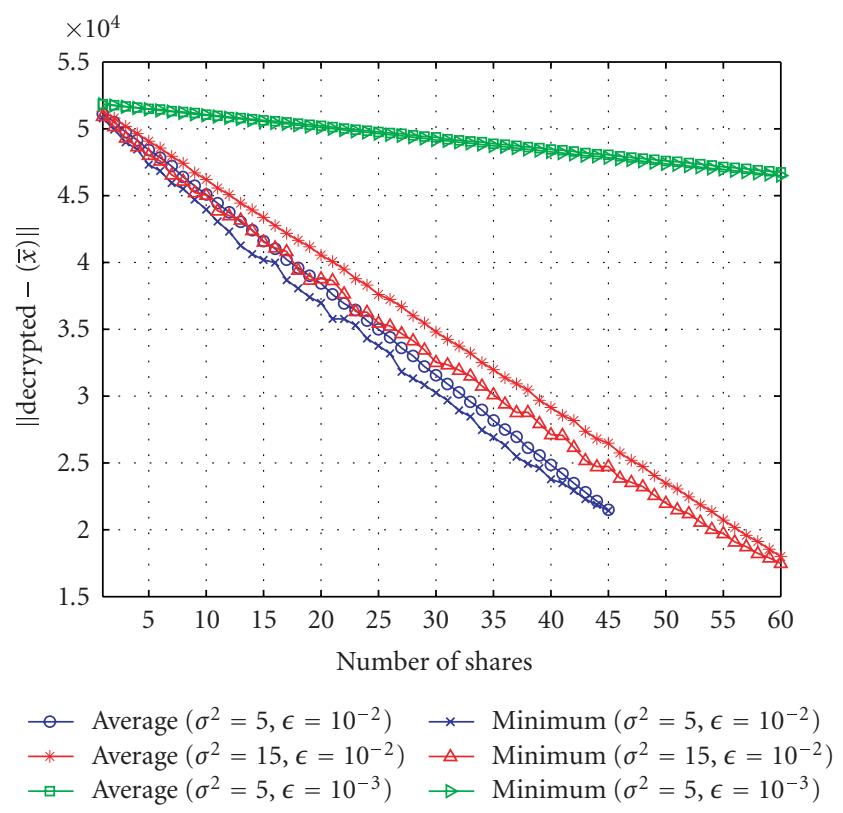

(a)

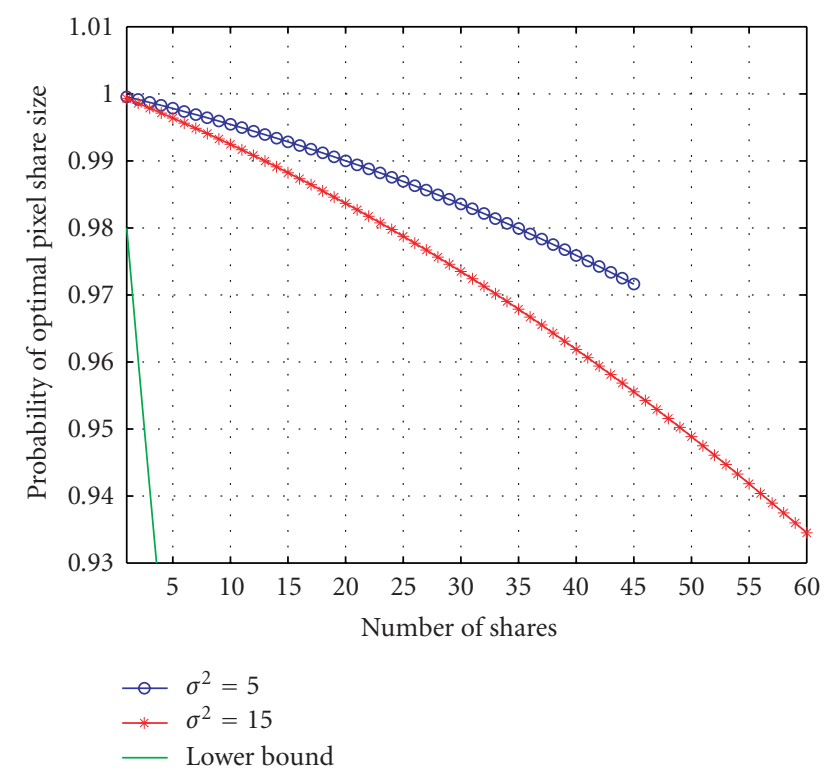

(b)

Figure 13: (a) $\left\|\mathbf{x}_{0}+\sum_{k=0}^{n} \mathbf{u}_{k}-\overline{\mathbf{x}}\right\|$ as a function of the number of shares $n$; (b) $\operatorname{Pr}\left\{\sum_{k=0}^{n}\left|u_{k}\right| \leq\left|\bar{x}-x_{0}\right|\right\}$ as a function of the number of shares $n$.

dimensions are approximately as those used in the simulations presented here in Figures 11, 12, and 13.

\section{REFERENCES}

[1] J. Wickramasuriya, M. Datt, S. Mehrotra, and N. Venkatasubramanian, "Privacy protecting data collection in media spaces," in Proceedings of the 12th ACM International Conference on Multimedia, pp. 48-55, New York, NY, USA, October 2004.

[2] D. Agathangelou, B. P. L. Lo, J. L. Wang, and G.-Z. Yang, "Selfconfiguring video-sensor networks," in Proceedings of the $3 \mathrm{rd}$ International Conference on Pervasive Computing, pp. 29-32, Munich, Germany, May 2005.
[3] G. Kogut, M. Blackburn, and H. R. Everett, "Using video sensor networks to command and control unmanned ground vehicles," in Proceedings of AUVSI Unmanned Systems in International Security, London, UK, September 2003.

[4] D. Kundur and W. Luh, "Multimedia sensor networks," in Encyclopedia of Multimedia, p. TBD, Springer, New York, NY, USA, 2006.

[5] M. Gerla and K. Xu, "Multimedia streaming in large-scale sensor networks with mobile swarms," ACM SIGMOD Record, vol. 32, no. 4, pp. 72-76, 2003.

[6] W.-C. Feng, J. Walpole, W.-C. Feng, and C. Pu, "Moving towards massively scalable video-based sensor networks," in Proceedings of Workshop on New Visions for Large-Scale Networks: Research and Applications, Washington, DC, USA, March 2001. 
[7] W.-C. Feng, B. Code, E. Kaiser, M. Shea, W.-C. Feng, and L. Bavoil, "Panoptes: scalable low-power video sensor networking technologies," in Proceedings of the ACM International Multimedia Conference, pp. 562-571, Berkeley, Calif, USA, November 2003.

[8] R. Holman, J. Stanley, and T. Özkan-Haller, "Applying video sensor networks to nearshore environment monitoring," IEEE Pervasive Computing, vol. 2, no. 4, pp. 14-21, 2003.

[9] A. Basharat, N. Catbas, and M. Shah, "A framework for intelligent sensor network with video camera for structural health monitoring of bridges," in Proceedings of 3rd IEEE International Conference on Pervasive Computing and Communications Workshops (PERCOM '05), pp. 385-389, Kauai Island, Hawaii, USA, March 2005.

[10] K. Obraczka, R. Manduchi, and J. J. Garcia-Luna-Aveces, "Managing the information flow in visual sensor networks," in Proceedings of 5th International Symposium on Wireless Personal Multimedia Communications (WPMC '02), vol. 3, pp. 1177-1181, Honolulu, Hawaii, USA, October 2002.

[11] J. Pan, Y. T. Hou, L. Cai, Y. Shi, and S. X. Shen, "Locating basestations for video sensor networks," in Proceedings of 58th IEEE Vehicular Technology Conference (VTC '04), vol. 5, pp. 30003004, Orlando, Fla, USA, October 2003.

[12] D. A. Fidaleo, H.-A. Nguyen, and M. Trivedi, "The networked sensor tapestry (NeST): a privacy enhanced software architecture for interactive analysis of data in video-sensor networks," in Proceedings of the ACM 2nd International Workshop on Video Sureveillance and Sensor Networks (VSSN '04), pp. 46-53, New York, NY, USA, October 2004.

[13] D. R. Stinson, Cryptography: Theory and Practice, Chapman \& Hall, New York, NY, USA, 1st edition, 1995.

[14] L. Tang, "Methods for encrypting and decrypting MPEG video data efficiently," in Proceedings of the 4th ACM International Conference on Multimedia, pp. 219-229, Boston, Mass, USA, November 1996.

[15] L. Qiao and K. Nahrstedt, "Comparison of MPEG encryption algorithms," Computers and Graphics, vol. 22, no. 4, pp. 437448, 1998.

[16] C. Shi and B. K. Bhargava, "A fast MPEG video encryption algorithm," in Proceedings of the 6th ACM International Conference on Multimedia, pp. 81-88, Bristol, England, September 1998.

[17] G. R. Blakley, "Safeguarding cryptographic keys," in Proceedings of the AFIPS 1979 National Computer Conference (NCC '79), vol. 48, pp. 313-317, Arlington, Va, USA, June 1979.

[18] A. Shamir, "How to share a secret," Communications of the ACM, vol. 22, no. 11, pp. 612-613, 1979.

[19] Z. Liu, D. Peng, Y. Zheng, and J. Liu, "Communication protection in IP-based video surveillance systems," in Proceedings of 7th IEEE International Symposium on Multimedia (ISM '05), pp. 69-78, Irvine, Calif, USA, December 2005.

[20] M. Naor and A. Shamir, "Visual cryptography," in Proceedings of Advances in Cryptology - EUROCRYPT'94, Workshop on the Theory and Application of Cryptographic Techniques, pp. 1-12, Perugia, Italy, May 1995.

[21] G. Ateniese, C. Blundo, A. De Santis, and D. R. Stinson, "Visual cryptography for general access structures," Information and Computation, vol. 129, no. 2, pp. 86-106, 1996.

[22] R. Ito, H. Kuwakado, and H. Tanaka, "Image size invariant visual cryptography," IEICE Transactions on Fundamentals of Electronics, Communications and Computer Science, vol. E82A, no. 10, pp. 2172-2177, 1999.
[23] C.-C. Lin and W.-H. Tsai, "Secret image sharing with capability of share data reduction," Optical Engineering, vol. 42, no. 8, pp. 2340-2345, 2003.

[24] Z. Xiong, A. D. Liveris, and S. Cheng, "Distributed source coding for sensor networks," IEEE Signal Processing Magazine, vol. 21, no. 5, pp. 80-94, 2004.

[25] A. Postma, W. de Boer, A. Helme, and G. Smit, "Distributed encryption and decryption algorithms," Memoranda Informatica 96-20, University of Twente, Enschede, The Netherlands, December 1996.

[26] A. Postma, Classes of Byzantine fault-tolerant algorithms for dependable distributed systems, Ph.D. thesis, University of Twente, Enschede, The Netherlands, 1998.

[27] W. Luh and D. Kundur, "Distributed privacy for visual sensor networks via Markov shares," in Proceedings of 2nd IEEE Workshop on Dependability and Security in Sensor Networks and Systems (DSSNS '06), pp. 23-34, Columbia, Md, USA, April 2006.

[28] A. Czarlinska and D. Kundur, "Distributed actuation attacks in wireless sensor networks: implications and countermeasures," in Proceedings of 2nd IEEE Workshop on Dependability and Security in Sensor Networks and Systems (DSSNS '06), pp. 3-12, Columbia, Md, USA, April 2006.

[29] T. A. Berson, "Failure of the McEliece public-key cryptosystem under message-resend and related-message conditions," in Advances in Cryptology-Proceedings of Crypto '97, B. Kaliski, Ed., vol. 1294 of Lecture Notes in Computer Science, pp. 213220, Springer, New York, NY, USA, 1997.

[30] Y. Q. Shi and H. Sun, Image and Video Compression for Multimedia Engineering: Fundamentals, Algorithms, and Standards, CRC Press, Boca Raton, Fla, USA, 2003.

[31] A. J. Menezes, P. C. van Oorschot, and S. A. Vanstone, Handbook of Applied Cryptography, CRC Press, Boca Raton, Fla, USA, 1st edition, 1996.

[32] U. N. Okorafor and D. Kundur, "Efficient routing protocols for a free space optical sensor network," in Proceedings of 2nd IEEE International Conference on Mobile Adhoc and Sensor Systems Conference, pp. 251-258, Washington, DC, USA, November 2005.

[33] D. Kundur, W. Luh, and U. Okorafor, "Security and rights management for multimedia sensor networks," in Multimedia Security Technologies for Digital Rights Management, Elsevier, New York, NY, USA, 2006.

[34] M. Vidyasagar, Nonlinear Systems Analysis, Prentice-Hall, Englewood Cliffs, NJ, USA, 2nd edition, 1993.

[35] R. M. Karp, "Reducibility among combinatorial problems," in Complexity of Computer Computations, R. E. Miller and J. W. Thatcher, Eds., pp. 85-104, Plenum Press, New York, NY, USA, 1972.

William Luh received the B.A.S. degree in computer engineering in 2002 from the University of Toronto, Canada, and the M.S. degree in electrical engineering in 2004 from Texas A\&M University. He is currently pursuing his Ph.D. degree in electrical engineering at Texas A\&M University under Dr. Deepa Kundur. His research interests include multimedia and sensor network security, digital rights management, watermark-

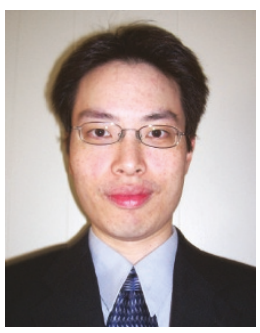
ing/fingerprinting, and steganography. 
Deepa Kundur received the B.A.S., M.A.S., and Ph.D. degrees all in electrical \& computer engineering in 1993, 1995, and 1999, respectively, from the University of Toronto, Canada. In January 2003, she joined the Department of Electrical \& Computer Engineering at Texas A\&M University where she leads the SeMANTIC (Sensor Media Algorithms \& Networking for Trusted Intelligent Computing) Research Group of the Wire-

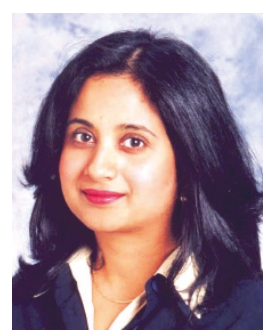
less Communications Laboratory. Before joining Texas A\&M, she was an Assistant Professor in the Department of Electrical and Computer Engineering at the University of Toronto where she was the Bell Canada Junior Chair-holder in Multimedia and an Associate Member of the Nortel Institute for Telecommunications. Her research interests include security and privacy for scalar and broadband sensor networks, multimedia security, digital rights management, steganalysis for computer forensics, and dynamical systems theory. She has given tutorials in the area of information security at ICME-2003 and Globecom-2003, and was a Guest Editor of the June 2004 Proceedings of the IEEE Special Issue on Enabling Security Technologies for Digital Rights Management. She currently serves as the Vice-Chair for the Security Interest Group of the IEEE Multimedia Communications Technical Committee and is an Associate Editor for the IEEE Communication Letters.

Takis Zourntos received the B.A.S., M.A.S., and Ph.D. degrees from the University of Toronto, Canada. His research interests are in the areas of nonlinear control and system theory, analog computation for robotics and optimization and integrated circuit implementation. He is currently an Assistant Professor with the Department of Electrical and Computer Engineering at Texas A\&M

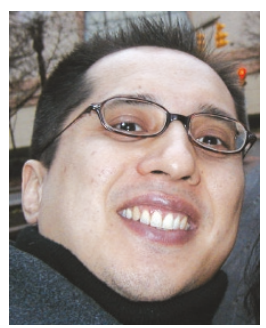
University, USA. 